\title{
Role of noise operators on two-photon correlations in an extended coherent Raman medium
}

\author{
C. H. Raymond Ooi ${ }^{1,2,3,4, *}$ and M. Suhail Zubairy ${ }^{3,5}$ \\ ${ }^{1}$ Department of Physics, Korea Advanced Institute of Science and Technology, Daejeon, 305-701 Korea \\ ${ }^{2}$ Max-Planck-Institut für Quantenoptik, D-85748, Garching, Germany \\ ${ }^{3}$ Institute for Quantum Studies and Department of Physics, Texas A\&M University, Texas 77843-4242, USA \\ ${ }^{4}$ Princeton Institute for the Science and Technology of Materials and Department of Mechanical \& Aerospace Engineering, \\ Princeton University, New Jersey 08544, USA \\ ${ }^{5}$ Texas A\&M University at Qatar, Education City, P.O. Box 23874, Doha, Qatar
}

(Received 8 January 2007; published 29 May 2007)

\begin{abstract}
An extended medium driven in a double Raman configuration generates Stokes and anti-Stokes fields that are described by coupled parametric oscillator equations with solutions that depend on input boundary operators and quantum noise operators. We identify the conditions where the boundary operators can be the substitute to the noise operators for describing two-photon cross correlation in forward and backward geometries. These conditions include short sample and small decoherence between ground states $\gamma_{b c}$, and they are fulfilled by the spontaneous Raman electromagnetic induced transparency scheme (weak pump with large detuning). We verify the correspondence between the results from boundary and noise operators analytically and show that the correlation due to the boundary operators is typically smaller than that due to the noise operators. In general the noise operators are required to obtain the correct correlation, especially when the control laser field is weak and $\gamma_{b c}$ is finite. Explanations for the findings are given based on the physics represented by the boundary operators and noise operators. Similar conclusions are obtained for the Stokes and anti-Stokes intensities.
\end{abstract}

DOI: 10.1103/PhysRevA.75.053822

PACS number(s): 42.50.Lc, 42.50.Dv, 03.65.Yz, 42.50.-p

\section{INTRODUCTION}

In the previous paper I (Ref. [1]), we have derived analytical expressions for two-photon correlations for a double Raman scheme with counterpropagating (backward) geometry and showed that the cross correlation of anti-Stokes to Stokes photons $G_{a s}^{(2)}$ computed using both noise operators and boundary operators is able to give good quantitative agreement with experimental data of Ref. [2]. Here, we extend the theory to copropagating (forward) geometry [Fig. 1(a)] and focus on comparing the results using the boundary operators and those using the noise operators.

Theory which neglects the noise operators [3], has been widely used for studying the generation of photon pairs in parametric amplifiers [4], Raman schemes [5], and optical fibers [6]. This simpler theoretical approach is an approximation which avoids all the complications associated with the noise operators. Recent interests in the generation of entangled photon pairs for quantum communications have spurred a lot of works on parametric amplifiers based on the $\chi^{(2)}$ parametric process. Although it is not always possible to neglect the noise operators, many literature and works have employed the noiseless approach to describe a variety of quantum phenomena [7], such as the generation of squeezed light [8] and entangled states. Proper theoretical study of quantum correlation in the amplifier which includes quantum noise and spatial propagation is desirable, particularly for determining the role of quantum noise and the validity of the semiclassical theory.

We will show that the extensively used semiclassical

\footnotetext{
*Email address: bokooi73@yahoo.com
}

theory does not, in general, give the correct correlation profile for an extended medium (length $L$ ) composed of many atoms (with number density $N$ ) in the Raman-EIT (electromagnetic induced transparency) scheme [Fig. 1(a)] especially when decoherence $\gamma_{b c}$ between levels $b$ and $c$ is finite or the optical density $N L \sigma$ is large, where $\sigma$ is the scattering cross section for the anti-Stokes. The situations for its validity are summarized in Fig. 1(b). Only for certain parameters, particularly when $\gamma_{b c}=0$ and $N L \sigma$ is small, qualitatively correct correlation can be obtained without the noise operators, but simply using the boundary operators with the commutation relation of the field at the boundary. The results will be verified analytically and the underlying physical reasons will be explained.

Contrary to usual expectations, the contribution of the noise operators to the correlation is typically larger than that of boundary operators, especially for large optical density. We use the quantum Heisenberg-Langevin-Maxwell theory [1] to study the effects of quantum interference (via resonant control field), decoherence, and propagation (via optical density) on the role of noise operators and the nonclassical properties of photon pairs.

\section{PARAMETRIC EQUATIONS FOR FORWARD GEOMETRY}

The coupled parametric equations for forward geometry (copropagating Stokes and anti-Stokes) are [1]

$$
\left(\frac{\partial}{\partial z}+\mathcal{G}_{s}\right) \hat{S}+\mathcal{K}_{s} \hat{A}^{\dagger}=\bar{F}_{s}
$$




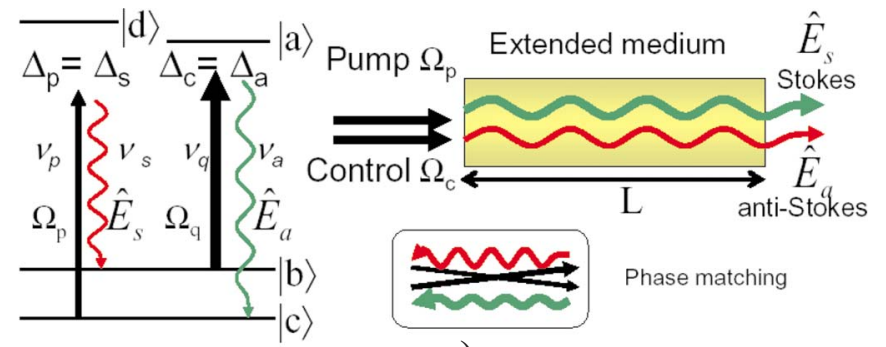

Weak field $\Omega$

\begin{tabular}{|c|c|c||c|c|c|c|}
\hline $\begin{array}{c}\text { Need noise } \\
\text { operators? }\end{array}$ & $\begin{array}{c}\text { Optically } \\
\text { thin }\end{array}$ & $\begin{array}{c}\text { Optically } \\
\text { thick }\end{array}$ & $\begin{array}{c}\text { Need noise } \\
\text { operators? }\end{array}$ & $\begin{array}{c}\text { Optically } \\
\text { thin }\end{array}$ & $\begin{array}{c}\text { Optically } \\
\text { thick }\end{array}$ \\
\hline$\gamma_{\mathrm{bc}}=0$ & No & Yes & $\gamma_{b c}=0$ & No & Mostly no \\
\hline$\gamma_{\mathrm{bc}}$ finite & Yes & Yes & $\gamma_{b c}$ finite & No & Perhaps no \\
\hline
\end{tabular}

b)

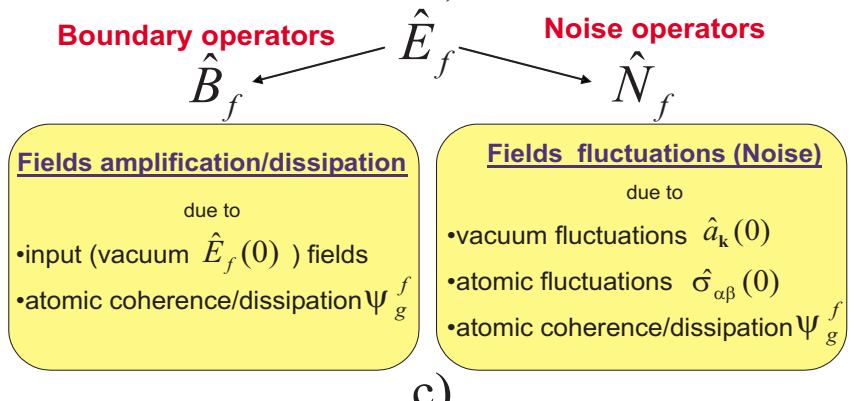

FIG. 1. (Color online) (a) Double Raman scheme for forward geometry (copropagating Stokes and anti-Stokes) where spontaneous Raman-EIT corresponds to $\Delta_{p}=\Delta_{s}>>\Omega_{p}$ and $\Delta_{c}=\Delta_{a}=0$. (b) Summary of the cases where the noise operators are required for correct cross-correlation $\left\langle\hat{E}_{s}^{\dagger}(t) \hat{E}_{a}^{\dagger}(t+\tau) \hat{E}_{a}(t+\tau) \hat{E}_{s}(t)\right\rangle$. (c) The roles and physical origins of the boundary operators and the noise operators.

$$
\left(\frac{\partial}{\partial z}+\mathcal{G}_{a}\right) \hat{A}^{\dagger}+\mathcal{K}_{a} \hat{S}=\bar{F}_{a}^{\dagger},
$$

with the effective noise operators

$$
\bar{F}_{s}=\sum_{x} M_{x} \hat{G}_{x}, \quad \bar{F}_{a}^{\dagger}=\sum_{x} N_{x} \hat{G}_{x}
$$

where $\hat{S}=g_{s} \hat{E}_{s}$ and $\hat{A}=g_{a} \hat{E}_{a}$ for the Stokes $\hat{E}_{s}$ and anti-Stokes fields $\hat{E}_{a}$, respectively, with $g_{s}=\mathcal{P}_{d b} / \hbar$ and $g_{a}=\mathcal{P}_{a c} / \hbar$ as the coupling strengths; $\quad \hat{G}_{x}=\int_{-\infty}^{\infty}\left[e^{i \theta_{x}(z, t)} \hat{F}_{x}(z, t)\right] e^{i \nu t} d t \quad(x$ $=a c, a d, b c, b d)$ are the Fourier transforms of the noise operators in the Heisenberg-Langevin equations with $\theta_{x}(z, t)$ $=k_{x} z-\nu_{x} t$ as the rapid varying phases. The microscopic expressions for $\mathcal{G}_{s}, \mathcal{K}_{s}, \mathcal{G}_{a}, \mathcal{K}_{a}, M_{x}$, and $N_{x}$ in Eqs. (1)-(3), are given in Appendix A.

The coupled equations are the same as for backward geometry except that $\partial / \partial z$ appears in Eq. (2) instead of $-\frac{\partial}{\partial z}$. The effective noise operators $\bar{F}_{s}$ and $\bar{F}_{a}^{\dagger}$ are the quantum seeds or driving "forces" to both fields due to quantum fluctuations of atomic ensembles in the extended medium. As we shall see, it incorporates dissipations via fluctuationdissipation theorem and the Einstein's relation [9].
For a sufficiently short sample, the spatial derivative in Eqs. (1) and (2) is negligible. These equations become identical to the familiar form

$$
\frac{d}{d t} \hat{\mathbf{a}}(t)=\left(\mathbf{G}-\frac{1}{2} \gamma\right) \hat{\mathbf{a}}(t)+\sqrt{\gamma} \hat{\mathbf{a}}_{i n}(t),
$$

used to describe single mode linear amplifier [10], and the input-output theory by Walls and Milburn [11], and Gardiner [12]. Here, the "input operator" $\hat{\mathbf{a}}_{\text {in }}$ plays the role of the noise operator and it satisfies the commutation relation for bosons. Of course, the solution of Eq. (4) would not contain any boundary operator. In vacuum, the input source $\sqrt{\gamma} \hat{\mathbf{a}}_{i n}(t)$ serves as the quantum seed.

\section{SOLUTIONS OF COUPLED EQUATIONS FOR OPERATORS}

The general solutions of Eqs. (1) and (2) are composed of two parts as

$$
\hat{E}_{f}(L, \nu)=\hat{B}_{f}(L, \nu)+\hat{N}_{f}(L, \nu),
$$

where $f=s$ (Stokes), $a$ (anti-Stokes). The term $\hat{B}_{f}$ contains the boundary operators $\hat{E}_{s}(z=0, \nu)$ and $\hat{E}_{a}^{\dagger}(z=0, \nu)$, and $\hat{N}_{f}$ contains the noise operators as shown in Eq. (3). The physical significance of the operators is summarized in Fig. 1(c). Those combined solutions include the laser fields to all orders and finite populations in upper levels beyond the adiabatic approximation. Solutions without $\hat{N}_{f}$ are considered as semiclassical. We compare the cross correlation computed from the solutions with the noise operators, solutions without the noise operators, and solutions with both noise and boundary operators.

\section{A. Noise parts}

The noise parts in the solutions, i.e., $\hat{N}_{s}(L, \nu)$ and $\hat{N}_{a}^{\dagger}(L, \nu)$ are written as convoluted spatial integrals of the noise operators

$$
\begin{aligned}
& \hat{N}_{s}(L, \nu)=\frac{1}{g_{s}} \int_{0}^{L}\left[\psi_{s}^{s}(\xi, \nu) \bar{F}_{s}(z, \nu)+\psi_{a}^{s}(\xi, \nu) \bar{F}_{a}^{\dagger}(z, \nu)\right] d z, \\
& \hat{N}_{a}^{\dagger}(L, \nu)=\frac{1}{g_{a}^{*}} \int_{0}^{L}\left[\psi_{s}^{a}(\xi, \nu) \bar{F}_{s}(z, \nu)+\psi_{a}^{a}(\xi, \nu) \bar{F}_{a}^{\dagger}(z, \nu)\right] d z,
\end{aligned}
$$

with the response functions to the noise sources $\bar{F}_{s}$ and $\bar{F}_{a}^{\dagger}$ that are spatially nonlocal

$$
\begin{gathered}
\psi_{s}^{s}(x, \nu)=\Xi_{q}(x)-\Xi(x) \mathcal{G}_{a}, \\
\psi_{a}^{s}(x, \nu)=\Xi(x) \mathcal{K}_{s}, \\
\psi_{s}^{a}(x, \nu)=\Xi(x) \mathcal{K}_{a},
\end{gathered}
$$




$$
\psi_{a}^{a}(x, \nu)=\Xi_{q}(x)-\Xi(x) \mathcal{G}_{s},
$$

where $\xi=L-z$. The spatially dependent oscillatory functions are

$$
\begin{gathered}
\Xi_{q}(x)=\frac{q_{+} e^{-q_{+} x}-q_{-} e^{-q_{-} x}}{q_{+}-q_{-}}, \\
\Xi(x)=\frac{e^{-q_{+} x}-e^{-q_{-} x}}{q_{+}-q_{-}},
\end{gathered}
$$

with $q_{ \pm}=\alpha_{+} \pm \beta, \alpha_{+}=\frac{1}{2}\left(\mathcal{G}_{a}+\mathcal{G}_{s}\right)$ as the total gain (loss) and $\beta=\sqrt{\alpha_{+}{ }^{2}+\mathcal{K}_{s} \mathcal{K}_{a}-G_{s} \mathcal{G}_{a}}$ governs the oscillation strength.

In Eqs. (6) and (7), the quantum noise operators act as the seeds for the amplified quantum fields $\hat{E}_{f}$. Please note that the coefficients $q_{ \pm}$depend on the coupling constants $g_{f}$. Thus, in the limit of small sample length $q_{+} x \rightarrow 0$, the solutions Eqs. (6) and (7) become independent of $g_{f}$ and hence the dipole matrix elements.

\section{B. Boundary parts}

The $\hat{B}_{s}(L, \nu)$ and $\hat{B}_{a}(L, \nu)$ are the solutions of Eqs. (1) and (2) at the output boundary $z=L$, do not depend on the noise operators, and they are expressed as a combination of the boundary input operators or vacuum field operators $\hat{E}_{s}(0, \nu)$ and $\hat{E}_{a}^{\dagger}(0, \nu)$,

$$
\begin{aligned}
& \hat{B}_{s}(L, \nu)=\psi_{s}^{s}(L, \nu) \hat{E}_{s}(0, \nu)+\psi_{a}^{s}(L, \nu) \frac{g_{a}^{*}}{g_{s}} \hat{E}_{a}^{\dagger}(0, \nu), \\
& \hat{B}_{a}^{\dagger}(L, \nu)=\psi_{a}^{a}(L, \nu) \hat{E}_{a}^{\dagger}(0, \nu)+\psi_{s}^{a}(L, \nu) \frac{g_{s}}{g_{a}^{*}} \hat{E}_{s}(0, \nu),
\end{aligned}
$$

where $\psi_{f}^{g}(L, \nu)(g, f=s, a)$ are defined by Eqs. (8)-(11) with $x=L$.

The solution $\hat{B}_{s}(L, \nu)$ contains the cross seeding antiStokes operator at the boundary $\hat{E}_{a}^{\dagger}(0, \nu)$ in addition to the Stokes $\hat{E}_{s}(0, \nu)$, and similarly for the solution of the antiStokes field operator. In the semiclassical solutions, the noise operators are neglected and the boundary field operators become the necessary seeds for the buildup of both the Stokes and anti-Stokes signals.

\section{Field commutation relation for vacuum fields}

The macroscopic quantum field can be expanded in terms of normal modes $u_{f}(z, \nu)$,

$$
\hat{E}_{f}(z, \nu)=\mathcal{E}_{f o} u_{f}(z, \nu) \hat{a}_{f}(\nu),
$$

where $\mathcal{E}_{f o}=\sqrt{\frac{\hbar v_{f}}{2 \varepsilon_{o} \mathcal{V}}}$ are the amplitudes, $f=s, a$, and

$$
u_{f}(z, \nu)=e^{i k(\nu) z}+S(z, \nu)
$$

are the normal mode functions. The first term is the solution of the homogenous coupled equations (without noise operators) and $S(z, \nu)$ is the part of the solution due to the noise source. At the input boundary $z=0$, it is clear that $S(0, \nu)$ $=0$. Thus, we have the normal and antinormal products for the boundary operators,

$$
\begin{aligned}
& \left\langle\hat{E}_{f}^{\dagger}(0, \nu) \hat{E}_{f}\left(0, \nu^{\prime}\right)\right\rangle=\mathcal{E}_{f o}^{2}\left\langle\hat{a}_{f}^{\dagger}(\nu) \hat{a}_{f}\left(\nu^{\prime}\right)\right\rangle, \\
& \left\langle\hat{E}_{f}(0, \nu) \hat{E}_{f}^{\dagger}\left(0, \nu^{\prime}\right)\right\rangle=\mathcal{E}_{f o}^{2}\left\langle\hat{a}_{f}(\nu) \hat{a}_{f}^{\dagger}\left(\nu^{\prime}\right)\right\rangle,
\end{aligned}
$$

which gives the commutation relation for vacuum fields

$$
\left[\hat{E}_{f}(0, \nu), \hat{E}_{f}^{\dagger}\left(0, \nu^{\prime}\right)\right]=C_{f} \delta\left(\nu-\nu^{\prime}\right)
$$

where $C_{f}=\frac{\hbar \nu_{f} \pi}{\varepsilon_{o} A c}$ as derived in Appendix D of Ref. [1].

In the case where the input radiation is a coherent state $|\alpha\rangle$ such as a laser, $\left\langle\hat{a}_{f}^{\dagger}(\nu) \hat{a}_{f}\left(\nu^{\prime}\right)\right\rangle=\frac{2 \pi L}{c}|\alpha|^{2} \delta\left(\nu-\nu^{\prime}\right)$. For a thermal state we have $\left\langle\hat{a}_{f}^{\dagger}(\nu) \hat{a}_{f}\left(\nu^{\prime}\right)\right\rangle=2 \pi L / c \bar{n}_{f} \delta\left(\nu-\nu^{\prime}\right)$, where $\bar{n}_{f}=\left(e^{\beta \hbar v_{f}}-1\right)^{-1}$. In the experiment of Harris [14], $T$ $=150 \mu \mathrm{K}$ corresponds to $\bar{n}_{f} \simeq 0$ and only the antinormal ordered term is finite.

\section{Noise solutions for short sample}

We show the connection between the solutions with noise operators and with boundary operators. The key point is the same functional form of the noise coefficients in Eqs. (6) and (7) and the boundary coefficients in Eqs. (14) and (15) [15]. In the limit of short sample $L q_{ \pm}<<1$, the nonlocal spatial dependence can be neglected, i.e.,

$$
\psi_{g}^{f}(\xi, \nu) \simeq \psi_{g}^{f}(L, \nu) .
$$

The $\psi_{g}^{f}$ coefficients in the solutions with noise operators are approximately local and can be factorized out from the spatial integrations

$$
\begin{aligned}
& \hat{N}_{s}(L, \nu) \simeq \psi_{s}^{s}(L, \nu) \int_{0}^{L} \bar{F}_{s}(z, \nu) \frac{d z}{g_{s}}+\psi_{a}^{s}(L, \nu) \int_{0}^{L} \bar{F}_{a}^{\dagger}(z, \nu) \frac{d z}{g_{s}}, \\
& \hat{N}_{a}^{\dagger}(L, \nu) \simeq \psi_{a}^{a}(L, \nu) \int_{0}^{z} \bar{F}_{a}^{\dagger}(z, \nu) \frac{d z}{g_{a}^{*}}+\psi_{s}^{a}(L, \nu) \int_{0}^{z} \bar{F}_{s}(z, \nu) \frac{d z}{g_{a}^{*}} .
\end{aligned}
$$

Now, we see that Eqs. (22) and (23) are of the same form as Eqs. (14) and (15), with $\int_{0}^{L} \bar{F}_{f}(z, \nu) d z$ in place of $\hat{E}_{f}(0, \nu)$. Even when the two terms (input field and noise parts) are of the same form, they may be very different numerically.

\section{E. Atomic commutation relation for noise operators}

From the similarity between the solutions with the boundary operators [Eqs. (14) and (15)] and the solutions with the noise operators [Eqs. (22) and (23)], it is tempting to ask whether the commutations $\left[\int_{0}^{L} \bar{F}_{f}(z, \nu) d z, \int_{0}^{L} \bar{F}_{f}\left(z^{\prime}, \nu^{\prime}\right) d z^{\prime}\right]$ look like Eq. (20). From Appendix C, we find

$$
\left\langle\left[\bar{F}_{f}(z, \nu), \bar{F}_{f}^{\dagger}\left(z^{\prime}, \nu^{\prime}\right)\right]\right\rangle=\overline{\mathcal{C}}_{f}(z, \nu) \delta\left(z-z^{\prime}\right) \delta\left(\nu-\nu^{\prime}\right),
$$

and hence 


$$
\left\langle\left[\int_{0}^{L} \bar{F}_{f}(z, \nu) d z, \int_{0}^{L} \bar{F}_{f}\left(z^{\prime}, \nu^{\prime}\right) d z^{\prime}\right]\right\rangle=\overline{\mathcal{C}}_{f}^{i n t}(\nu) \delta\left(\nu-\nu^{\prime}\right),
$$

where $\overline{\mathcal{C}}_{f}^{i n t}(\nu)=\int_{0}^{L} \overline{\mathcal{C}}_{f}(z, \nu) d z$.

Equation (25) is the atomic noise commutation relation which relates quantum fluctuations and dissipations of atomic ensembles. The commutation relation is of the same form as the field commutation relation Eq. (20) for the boundary operators $\left[\hat{E}_{f}(0, \nu), \hat{E}_{f}^{\dagger}\left(0, \nu^{\prime}\right)\right]=C_{f} \delta\left(\nu-\nu^{\prime}\right)$. But we cannot conclude that the solutions with the noise operators are identical to the solutions with boundary operators unless the $\nu$ dependence in $\overline{\mathcal{C}}_{f}^{i n t}(\nu)$ becomes unimportant, as we show in Sec. V C.

\section{TWO-PHOTON CORRELATION}

We now use the solutions for the field operators, Eqs. (5)-(7), (14), and (15) to compute the cross correlations between the Stokes and anti-Stokes fields $G_{a s}^{(2)}$ and $G_{s a}^{(2)}$, and the self-correlations $G_{s s}^{(2)}$ and $G_{a a}^{(2)}$.

\section{A. Correlation due to thermal radiation}

First, let us compute the correlation of the radiation in the thermal background without the atomic medium. The correlation can be calculated as

$$
\begin{aligned}
G_{\text {the }}^{(2)}(t, \tau) & =\left|\left\langle E^{\dagger}(t+\tau) E(t)\right\rangle\right|^{2}+\left\langle\mathcal{E}^{\dagger}(t+\tau) \mathcal{E}(t+\tau)\right\rangle\left\langle\mathcal{E}^{\dagger}(t) \mathcal{E}(t)\right\rangle \\
& =\left.\left.\left|\sum_{\mathbf{k}}\right| g_{\mathbf{k}}\right|^{2} \bar{n}_{\mathbf{k}} e^{-i \nu_{\mathbf{k}} \tau}\right|^{2}+\left.\left.\left|\sum_{\mathbf{k}}\right| g_{\mathbf{k}}\right|^{2} \bar{n}_{\mathbf{k}}\right|^{2},
\end{aligned}
$$

where $\bar{n}_{\mathbf{k}}=\left\langle a_{\mathbf{k}}^{\dagger} a_{\mathbf{k}}\right\rangle=\left(e^{\beta \hbar \nu_{\mathbf{k}}}-1\right)^{-1}$ is the mean number of photons in mode $\mathbf{k}$. We find that the multimode thermal radiation is also super-Poissonian with normalized correlation $g_{\text {the }}^{(2)}(0)$ $=2$ and bunching $g_{\text {the }}^{(2)}(\tau)<2$, in contrast to the single mode thermal state where $g_{\text {the }}^{(2)}(\tau)=2$ for all $\tau$.

However, the quantity of interest to experiment is the twophoton cross-correlation function $G_{a s}^{(2)} \doteq\left\langle\hat{E}_{s}^{\dagger}(t) \hat{E}_{a}^{\dagger}(t+\tau) \hat{E}_{a}(t\right.$ $\left.+\tau) \hat{E}_{s}(t)\right\rangle$, which quantifies to what extent the macroscopic anti-Stokes field is correlated to the preceding macroscopic Stokes field. The contribution of the thermal background to the correlation is taken care of by the solutions Eqs. (14) and (15). This can be seen by omitting the noise contribution to Eqs. (16) and (17),

$$
\hat{E}_{f}(z, \nu) \rightarrow \mathcal{E}_{f}(z, \nu)=\hat{E}_{f}(z, \nu)=\mathcal{E}_{f o}(\nu) e^{i k(\nu) z} \hat{a}_{f}(\nu) .
$$

By using the fact that the two modes are independent $\left\langle a_{s}^{\dagger} a_{a}\right\rangle=0$ we have

$$
\begin{aligned}
G_{a s 0}^{(2)} & \doteq\left\langle\mathcal{E}_{s}^{\dagger}(t) \mathcal{E}_{a}^{\dagger}(t+\tau) \mathcal{E}_{a}(t+\tau) \mathcal{E}_{s}(t)\right\rangle \\
& =\left\langle\mathcal{E}_{a}^{\dagger}(t+\tau) \mathcal{E}_{a}(t+\tau)\right\rangle\left\langle\mathcal{E}_{s}^{\dagger}(t) \mathcal{E}_{s}(t)\right\rangle \\
& =\left|g_{s}\right|^{2} \bar{n}_{s}\left|g_{a}\right|^{2} \bar{n}_{a},
\end{aligned}
$$

which is independent of delay $\tau$ since the thermal photons at Stokes and anti-Stokes frequencies are independent. There- fore, the normalized correlation corresponds to a coherent state $g_{0}^{(2)}(\tau)=1$, which is different from $g_{\text {the }}^{(2)}$. Thus, the correlation defined by Eq. (28) does not include the correlation of the thermal photons in the background. This is fine since we are only interested in the effects of thermal photons through the interaction with atomic medium and not through direct detection.

\section{B. Correlations with only boundary operators}

The solutions without noise operators Eqs. (14) and (15) give the two-photon cross-correlation function

$$
\begin{aligned}
G_{a s}^{(2) b}(L, \tau) & \doteq\left\langle\hat{B}_{s}^{\dagger}(L, t) \hat{B}_{a}^{\dagger}(L, t+\tau) \hat{B}_{a}(L, t+\tau) \hat{B}_{s}(L, t)\right\rangle \\
& =\left|\left\langle\hat{B}_{a}(L, \tau) \hat{B}_{s}(L)\right\rangle\right|^{2}+\mathcal{I}_{a}^{b}(L) \mathcal{I}_{s}^{b}(L),
\end{aligned}
$$

and similarly the reverse correlation

$$
\begin{aligned}
G_{s a}^{(2) b}(L, \tau) & \doteq\left\langle\hat{B}_{a}^{\dagger}(L, t) \hat{B}_{s}^{\dagger}(L, t+\tau) \hat{B}_{s}(L, t+\tau) \hat{B}_{a}(L, t)\right\rangle \\
& =\left|\left\langle\hat{B}_{s}(L, \tau) \hat{B}_{a}(L)\right\rangle\right|^{2}+\mathcal{I}_{s}^{b}(L) \mathcal{I}_{a}^{b}(L) .
\end{aligned}
$$

The cross-correlation amplitude $\left\langle\hat{B}_{a}(L, \tau) \hat{B}_{s}(L)\right\rangle$, reversecorrelation amplitude $\left\langle\hat{B}_{s}(L, \tau) \hat{B}_{a}(L)\right\rangle$, and intensities $\mathcal{I}_{f}^{b}(L)$ are obtained by straightforward calculations using the solutions Eqs. (14) and (15), the commutation relation Eq. (20), and the inverse Fourier transforms $\left(\begin{array}{c}\hat{B}_{a}(t) \\ \hat{B}_{a}^{\dagger}(t)\end{array}\right)=\int_{-\infty}^{\infty}\left(\begin{array}{c}\hat{B}_{s}(\nu) \\ \hat{B}_{a}^{\dagger}(v)\end{array}\right) e^{-i \nu t} d \nu$. Those expressions are given by

$$
\begin{aligned}
\left\langle\hat{B}_{a}(L, \tau) \hat{B}_{s}(L)\right\rangle= & \int_{-\infty}^{\infty}\left[C_{a}\left(\bar{n}_{a}+1\right) \frac{g_{a}^{*}}{g_{s}} \psi_{a}^{a^{*}}(L, \nu) \psi_{a}^{s}(L, \nu)\right. \\
& \left.+C_{s} \bar{n}_{s} \frac{g_{s}^{*}}{g_{a}} \psi_{s}^{a^{*}}(L, \nu) \psi_{s}^{s}(L, \nu)\right] e^{i \nu \tau} \frac{d \nu}{2 \pi},
\end{aligned}
$$

$$
\begin{aligned}
\left\langle\hat{B}_{s}(L, \tau) \hat{B}_{a}(L)\right\rangle= & \int_{-\infty}^{\infty}\left[C_{a} \bar{n}_{a} \frac{g_{a}^{*}}{g_{s}} \psi_{a}^{a^{*}}(L, \nu) \psi_{a}^{s}(L, \nu)\right. \\
& \left.+C_{s}\left(\bar{n}_{s}+1\right) \frac{g_{s}^{*}}{g_{a}} \psi_{s}^{a^{*}}(L, \nu) \psi_{s}^{s}(L, \nu)\right] e^{-i \nu \tau} \frac{d \nu}{2 \pi},
\end{aligned}
$$

where $\bar{n}_{f}=\left(e^{\beta \hbar \nu_{f}}-1\right)^{-1}(f=s, a)$ are the mean photon numbers and $\mathcal{I}_{f}^{b}(L)=\mathcal{I}_{s}^{b}(L, \tau=0)$ are obtained from the self-correlation amplitudes

$$
\begin{aligned}
\mathcal{I}_{s}^{b}(L, \tau)= & \left\langle\hat{B}_{s}^{\dagger}(L, \tau) \hat{B}_{s}(L)\right\rangle \\
= & \int_{-\infty}^{\infty}\left[C_{a}\left(\bar{n}_{a}+1\right)\left|\frac{g_{a}^{*}}{g_{s}} \psi_{a}^{s}(L, \nu)\right|^{2}\right. \\
& \left.+C_{s} \bar{n}_{s}\left|\psi_{s}^{s}(L, \nu)\right|^{2}\right] e^{i \nu \tau} \frac{d \nu}{2 \pi},
\end{aligned}
$$




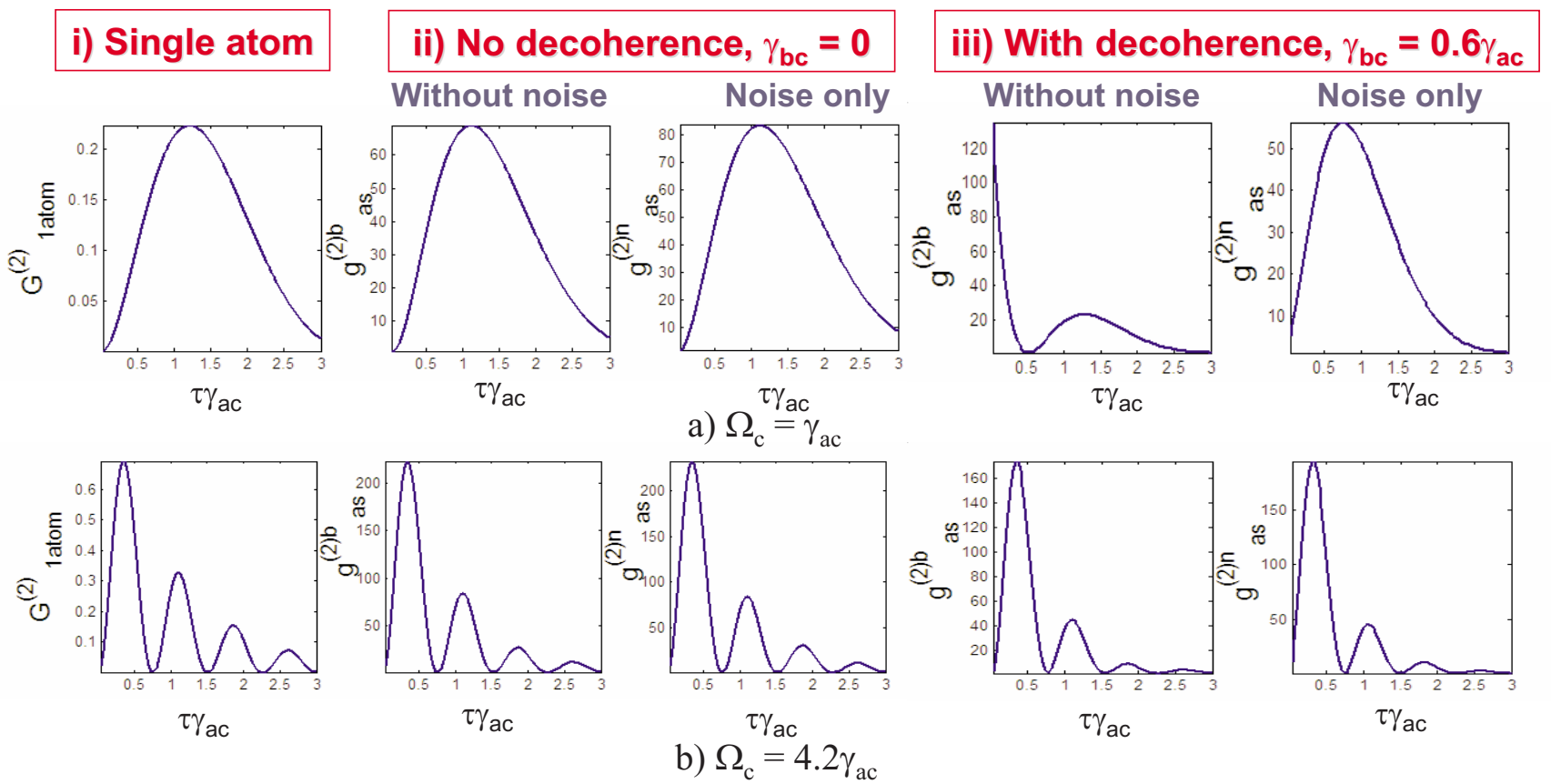

FIG. 2. (Color online) Correlations with relatively small dipole moment $\wp=5 \times 10^{-30} \mathrm{Cm}$ for three categories: (i) $G_{1}^{(2)}$ atom for single Raman-EIT atom, (ii) extended medium in forward geometry without decoherence $\gamma_{b c}=0$, and (iii) extended medium in forward geometry with decoherence $\gamma_{b c}=0.6 \gamma_{a c}$. The normalized correlation $g_{a s}^{(2) b}=G_{a s}^{(2) b}(L, \tau) / \mathcal{I}_{s}^{b}(L) \mathcal{I}_{a}^{b}(L)$ is obtained with a commutation relation for the operator at boundary but without the noise operator and $g_{a s}^{(2) n}=G_{a s}^{(2) n}(L, \tau) / \mathcal{I}_{s}(L) \mathcal{I}_{a}(L)$ is obtained with only noise operators and no zero boundary operators. Other parameters are $\Delta=-7.5 \gamma_{a c}, \Omega_{p}=0.2 \gamma_{a c}$, and $N \sigma L=0.528\left(L=1.5 \mathrm{~mm}\right.$ and $\left.N=8 \times 10^{16} \mathrm{~m}^{-3}\right)$. As the control field increases from (a) $\Omega_{c}=\gamma_{a c}$ to (b) $\Omega_{c}=4.2 \gamma_{a c}$, the correlation with the boundary operator gives the correct result, in close quantitative agreement with the normalized correlation using only noise operators.

$$
\begin{aligned}
\mathcal{I}_{a}^{b}(L, \tau)= & \left\langle\hat{B}_{a}^{\dagger}(L, \tau) \hat{B}_{a}(L)\right\rangle \\
= & \int_{-\infty}^{\infty}\left[C_{s}\left(\bar{n}_{s}+1\right)\left|\frac{g_{s}}{g_{a}^{*}} \psi_{s}^{a}(L, \nu)\right|^{2}\right. \\
& \left.+C_{a} \bar{n}_{a}\left|\psi_{a}^{a}(L, \nu)\right|^{2}\right] e^{-i \nu \tau} \frac{d \nu}{2 \pi} .
\end{aligned}
$$

The finiteness of the correlation amplitudes in Eqs. (31) and (32) is due to the noncommutivity of the input Stokes and anti-Stokes operators at the boundary, i.e., via $C_{f}$. In the absence of thermal photons $\bar{n}_{f}=0$, Eq. (31) depends only on $\psi_{a}^{s}(L, \nu)$ and $\psi_{a}^{a}(L, \nu)$. So, the cross correlation in $G_{a s}^{(2) b}$ is attributed to the boundary operator $\hat{E}_{a}(0, \nu)$ for the antiStokes. By the same arguments, the reverse correlation in $G_{s a}^{(2) b}$ is due to the boundary operator $\hat{E}_{s}(0, \nu)$ for the Stokes.

Equations (29) and (30) are used to compute the normalized correlations $g_{a s(s a)}^{(2) b}(L, \tau) \doteq G_{a s(s a)}^{(2) b}(L, \tau) / \mathcal{I}_{s}^{b}(L) \mathcal{I}_{a}^{b}(L)$ in Figs. 2-4.

\section{Correlations with only noise operators}

The cross and reverse correlations due to the noise operators alone are defined like Eqs. (29) and (30), respectively, except that $\hat{B}$ is replaced by $\hat{N}$,

$$
G_{a s}^{(2) n}(L, \tau)=\left|\left\langle\hat{N}_{a}(L, \tau) \hat{N}_{s}(L)\right\rangle\right|^{2}+\mathcal{I}_{s}^{n}(L) \mathcal{I}_{a}^{n}(L),
$$

$$
G_{s a}^{(2) n}(L, \tau)=\left|\left\langle\hat{N}_{s}(L, \tau) \hat{N}_{a}(L)\right\rangle\right|^{2}+\mathcal{I}_{s}^{n}(L) \mathcal{I}_{a}^{n}(L) .
$$

Here, the cross-correlation amplitudes $\left\langle\hat{N}_{a}(L, t\right.$ $\left.+\tau) \hat{N}_{s}(L, t)\right\rangle, \quad$ reverse-correlation amplitude $\left\langle\hat{N}_{s}(L, t\right.$ $\left.+\tau) \hat{N}_{a}(L, t)\right\rangle$, and the intensities $\mathcal{I}_{f}^{n}(L)$ due to the noise operators are given by [13]

$$
\begin{gathered}
\left\langle\hat{N}_{a}(L, \tau) \hat{N}_{s}(L)\right\rangle=e^{i \Delta k L} \frac{2 \pi}{A N} \int_{-\infty}^{\infty} e^{i \nu \tau} \Phi(L, \nu) d \nu, \\
\left\langle\hat{N}_{s}(L, \tau) \hat{N}_{a}(L)\right\rangle=e^{i \Delta k L} \frac{2 \pi}{A N} \int_{-\infty}^{\infty} e^{-i \nu \tau} \Psi(L, \nu) d \nu,
\end{gathered}
$$

with the phase mismatch $\Delta k$ and the intensities $\mathcal{I}_{f}^{n}(L)$ $=\mathcal{I}_{f}^{n}(L, \tau=0)$ are obtained from the self-correlation amplitudes

$$
\mathcal{I}_{s}^{n}(L, \tau)=\left\langle\hat{N}_{s}^{\dagger}(L, \tau) \hat{N}_{s}(L)\right\rangle=\frac{2 \pi}{A N} \int_{-\infty}^{\infty} e^{i \nu \tau} S(L, \nu) d \nu,
$$

$$
\mathcal{I}_{a}^{n}(L, \tau)=\left\langle\hat{N}_{a}^{\dagger}(L, \tau) \hat{N}_{a}(L)\right\rangle=\frac{2 \pi}{A N} \int_{-\infty}^{\infty} e^{-i \nu \tau} A(L, \nu) d \nu .
$$

Thus, the above correlation amplitudes are the Fourier transforms of the spectral functions 

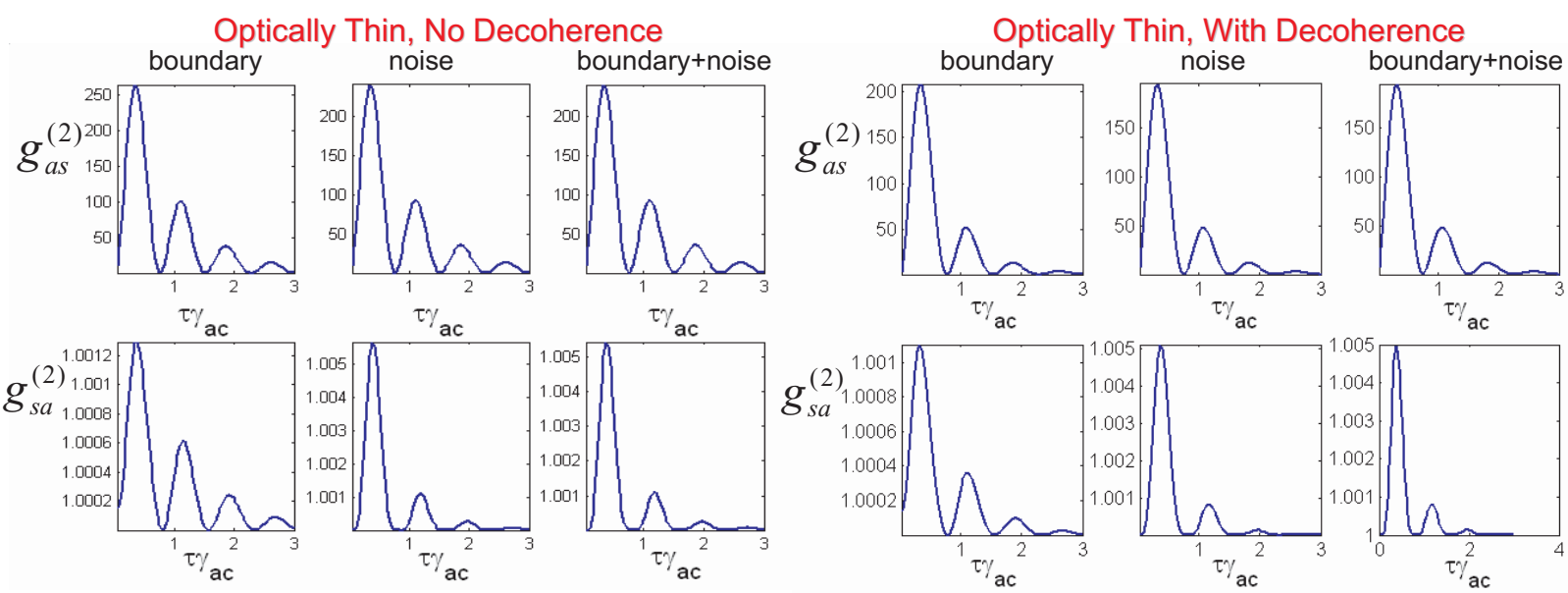

Optically Thick, No Decoherence
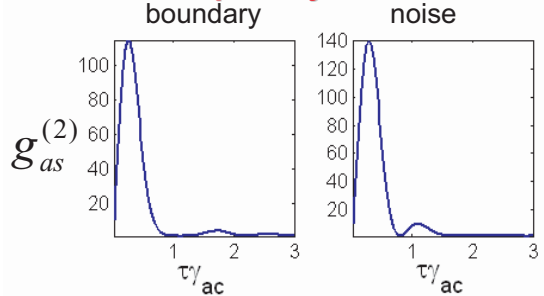

boundary+noise
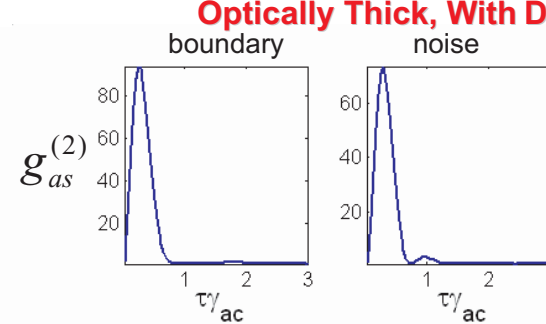

boundary+noise
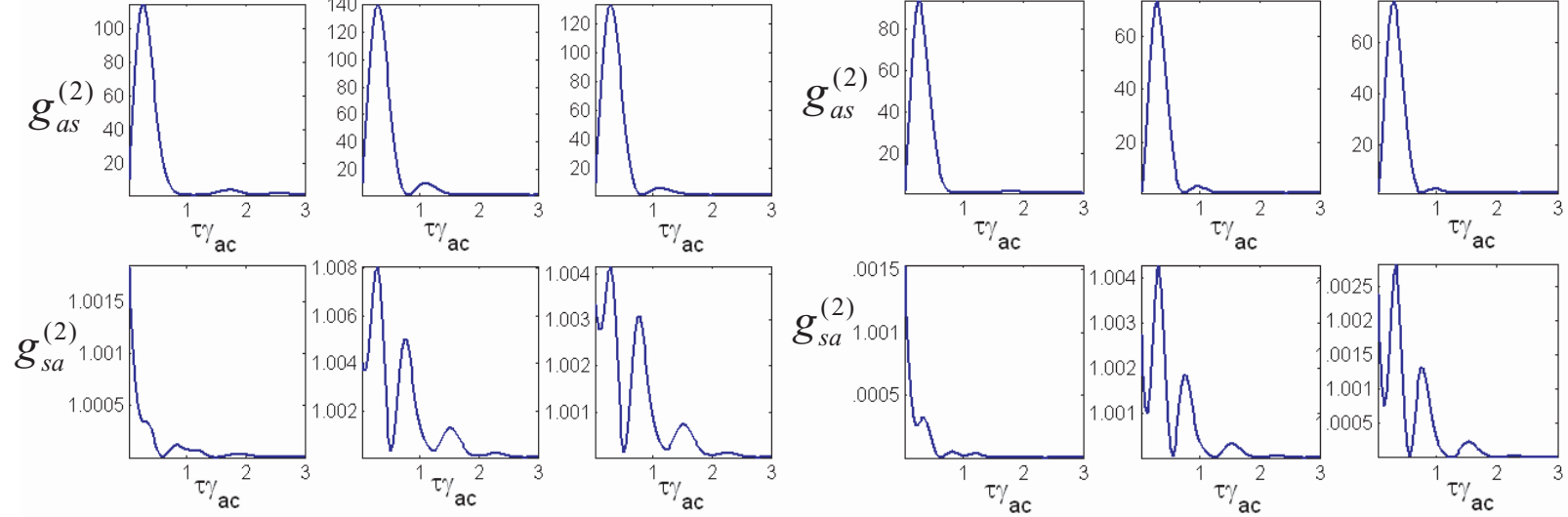

FIG. 3. (Color online) Cross correlation $g_{a s}^{(2)}$ and the reverse correlation $g_{s a}^{(2)}$ computed with boundary (without noise) operators, noise operators only, and both boundary and noise operators for forward geometry. The four cases involve, with decoherence $\gamma_{b c}=0.6 \gamma_{a c}$, no decoherence $\gamma_{b c}=0$, short sample $N \sigma L=1$, and long sample $N \sigma L=11$. Other parameters are $\Delta=-7.5 \gamma_{a c}, \Omega_{p}=0.4 \gamma_{a c}$, and $\Omega_{c}=4.2 \gamma_{a c}$. We have computed the entire results for backward geometry as well (based on Ref. [1]) and find that there is no noticeable difference from the forward case for the cross correlation and reverse correlation, even for a long sample.

$$
\begin{aligned}
& \Phi(L, \nu)=\sum_{x, x^{\prime}} \int_{0}^{L} 2 \widetilde{D}_{x, x^{\prime}}^{n}(z) C_{x}^{a^{*}}(\xi, \nu) C_{x^{\prime}}^{s}(\xi, \nu) d z, \\
& \Psi(L, \nu)=\sum_{x, x^{\prime}} \int_{0}^{L} 2 \widetilde{D}_{x, x^{\prime}}^{a n}(z) C_{x}^{s}(\xi, \nu) C_{x^{\prime}}^{a^{*}}(\xi, \nu) d z, \\
& S(L, \nu)=\sum_{x, x^{\prime}} \int_{0}^{L} 2 \widetilde{D}_{x, x^{\prime}}^{n}(z) C_{x}^{s^{*}}(\xi, \nu) C_{x^{\prime}}^{s}(\xi, \nu) d z, \\
& A(L, \nu)=\sum_{x, x^{\prime}} \int_{0}^{L} 2 \widetilde{D}_{x, x^{\prime}}^{a n}(z) C_{x}^{a}(\xi, \nu) C_{x^{\prime}}^{a^{*}}(\xi, \nu) d z,
\end{aligned}
$$

where $x, x^{\prime}=a c, a d, b c, b d$. The expressions for $2 \widetilde{D}_{x, x^{\prime}}^{n(a n)}(z, t)$ $\doteq 2 D_{x, x^{\prime}}^{n(a n)}(z, t) e^{-i \theta_{x}(z, t)} e^{i \theta_{x^{\prime}}(z, t)}$, the slowly varying normal (antinormal) ordered diffusion coefficients can be obtained from Einstein's relation [9] $\frac{d}{d t}\langle\hat{x} \hat{y}\rangle=2 D_{x, x^{\prime}}^{n(a n)}(z, t)+\left\langle\hat{x} \hat{A}_{y}\right\rangle+\left\langle\hat{A}_{x} \hat{y}\right\rangle$ and a pair of Heisenberg-Langevin equations $\frac{d}{d t} \hat{q}(z, t)=\hat{A}_{q}(z, t)$

$+\hat{F}_{q}(z, t), q=x, y$. Equations (35)-(44) imply that the amplified quantum fluctuations contribute to the two-photon correlation via the diffusion coefficients.

The $C_{x}^{f}$ coefficients $(f=s, a)$ in Eqs. (41)-(44) are defined as

$$
\begin{aligned}
C_{x}^{s}(\xi, \nu) & =\frac{1}{g_{s}}\left[\psi_{s}^{s}(\xi, \nu) M_{x}(\nu)+\psi_{a}^{s}(\xi, \nu) N_{x}(\nu)\right], \\
C_{x}^{a}(\xi, \nu) & =\frac{1}{g_{a}^{*}}\left[\psi_{a}^{a}(\xi, \nu) N_{x}(\nu)+\psi_{s}^{a}(\xi, \nu) M_{x}(\nu)\right],
\end{aligned}
$$

with $M_{x}(\nu)$ and $N_{x}(\nu)$ given by Eqs. (A5) and (A6).

The adjoint of Eq. (37) can also be expressed in an alternative form $\frac{2 \pi e^{i \Delta k L}}{A N} \Sigma_{x, x^{\prime}} \int_{0}^{L} 2 \widetilde{D}_{x, x^{\prime}}^{n}(z) \int_{-\infty}^{\infty} \widetilde{C}_{x}^{a^{*}}\left(\xi, t^{\prime}\right) \widetilde{C}_{x^{\prime}}^{s}(\xi, \tau$ $\left.-t^{\prime}\right) d t^{\prime} d z$ using the convolution theorem. If the spatial variations of the populations and the coherences are much slower than $\mathcal{G}_{f}, \mathcal{K}_{f}$, the diffusion coefficients $\widetilde{D}_{x, x^{\prime}}^{n(a n)}$ can be separated from the functions $C_{x}^{s}, C_{x}^{a}$ in the spatial integrals and Eqs. (41)-(44) can be integrated analytically. 

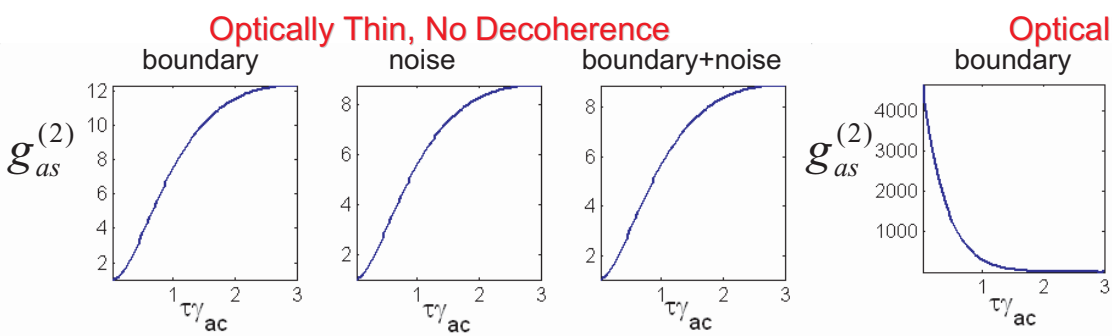

Optically Thin, With Decoherence
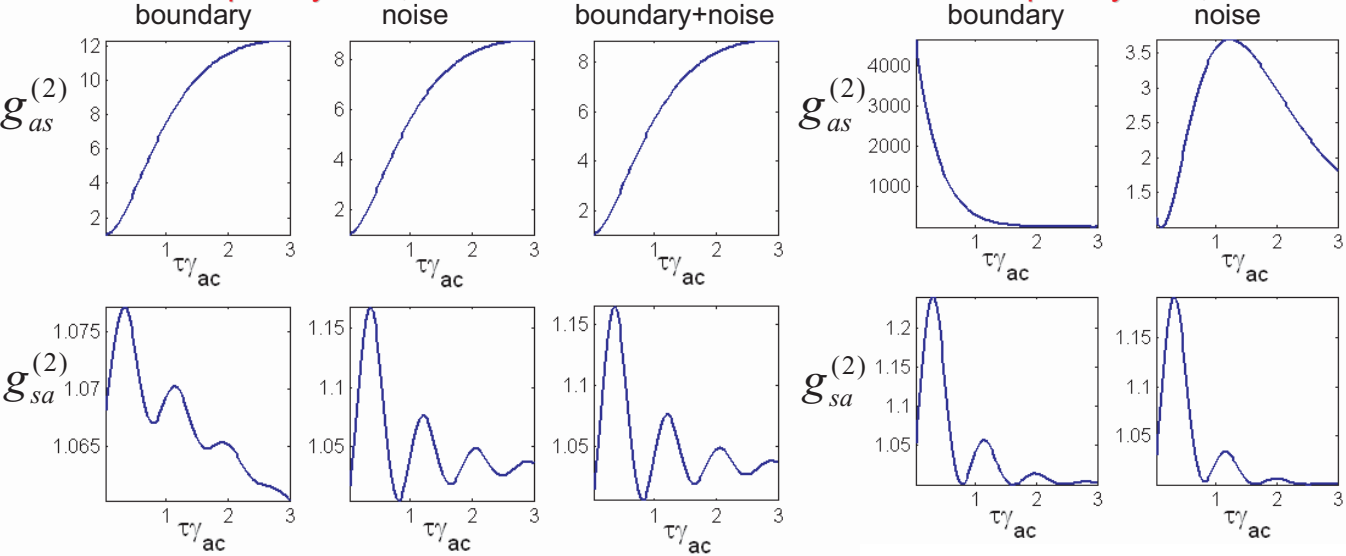

boundary+noise

ptically Thick, No Decoherence
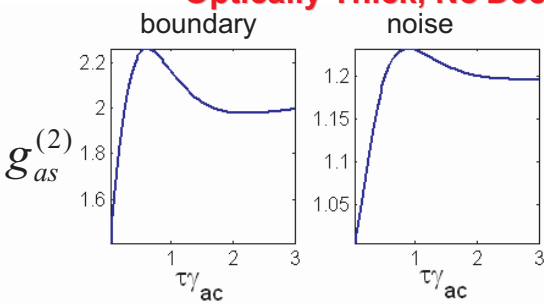

boundary+noise
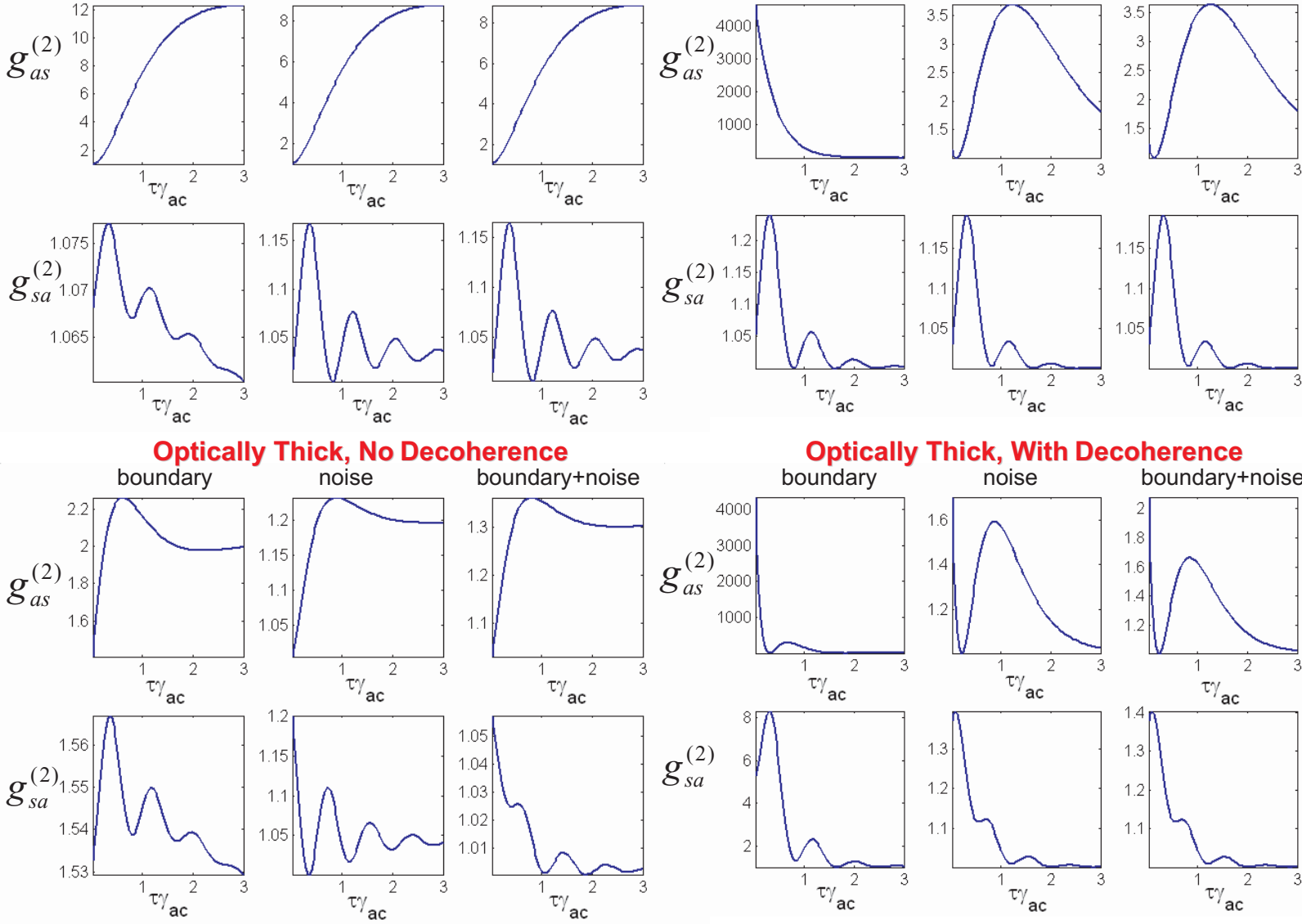

FIG. 4. (Color online) Same as Fig. 3 except with a smaller control Rabi frequency $\Omega_{c}=0.2 \gamma_{a c}$. Again, we do not show the entire results for backward geometry since they are indistinguishable from the above figures.

For comparison with $g_{a s(s a)}^{(2) b}$, the normalized correlations due to the noise operators only $g_{a s(s a)}^{(2) n}(L, \tau)$ $\doteq G_{a s(s a)}^{(2) n}(L, \tau) / \mathcal{I}_{s}^{n}(L) \mathcal{I}_{a}^{n}(L)$ are plotted in Figs. 2-4.

\section{Total cross correlation}

The total cross correlation $G_{a s}^{(2)}$ is obtained by summing up $G_{a s}^{(2) b}, G_{a s}^{(2) n}$, and the terms with combinations of the boundary operators and noise operators,

$$
\begin{aligned}
G_{a s}^{(2)}(L, \tau) & \equiv\left\langle\hat{E}_{s}^{\dagger}(L, t) \hat{E}_{a}^{\dagger}(L, t+\tau) \hat{E}_{a}(L, t+\tau) \hat{E}_{s}(L, t)\right\rangle \\
& =\left|\left\langle\hat{B}_{a}(L, \tau) \hat{B}_{s}(L)\right\rangle+\left\langle\hat{N}_{a}(L, \tau) \hat{N}_{s}(L)\right\rangle\right|^{2}+\mathcal{I}_{s}(L) \mathcal{I}_{a}(L),
\end{aligned}
$$

where the total intensities are $\mathcal{I}_{f}(L)=\mathcal{I}_{f}^{b}(L)+\mathcal{I}_{f}^{n}(L)$.

Similarly, we have the reverse correlation

$$
\begin{aligned}
G_{s a}^{(2)}(L, \tau) & \doteq\left\langle\hat{E}_{a}^{\dagger}(L, t) \hat{E}_{s}^{\dagger}(L, t+\tau) \hat{E}_{s}(L, t+\tau) \hat{E}_{a}(L, t)\right\rangle \\
& =\left|\left\langle\hat{B}_{s}(L, \tau) \hat{B}_{a}(L)\right\rangle+\left\langle\hat{N}_{s}(L, \tau) \hat{N}_{a}(L)\right\rangle\right|^{2}+\mathcal{I}_{s}(L) \mathcal{I}_{a}(L),
\end{aligned}
$$

and the self-correlations

$$
\begin{aligned}
G_{f f}^{(2)}(L, \tau) & \doteq\left\langle\hat{E}_{f}^{\dagger}(t) \hat{E}_{f}^{\dagger}(t+\tau) \hat{E}_{f}(t+\tau) \hat{E}_{f}(t)\right\rangle \\
& =\left|\mathcal{I}_{f}^{b}(L, \tau)+\mathcal{I}_{f}^{n}(L, \tau)\right|^{2}+\mathcal{I}_{f}(L)^{2} .
\end{aligned}
$$

The normalized total correlations that include both the boundary and noise operators can be computed from $g_{a s(s a)}^{(2)}(L, \tau) \doteq G_{a s(s a)}^{(2)}(L, \tau) / \mathcal{I}_{s}(L) \mathcal{I}_{a}(L)$.

\section{CORRESPONDENCE BETWEEN BOUNDARY AND NOISE PARTS}

In the limit where the decoherence vanishes $\gamma_{b c} \rightarrow 0$, large control field $\Omega_{c}$, and sufficiently short sample $q_{ \pm} L \ll 1\left(q_{ \pm}\right.$ $\left.\sim \alpha_{a} \sim g_{a} \kappa_{a} / \gamma_{a c} \sim N \sigma / 2\right)$, Fig. 2 shows that the normalized correlation $g_{a s}^{(2)}$ for the Raman-EIT scheme computed with boundary operators and with noise operators are in excellent quantitative agreement. This motivates further analysis to understand such a correspondence.

\section{A. Identical correlation for Raman-EIT and short sample}

Here, we show that the correlation Eq. (35) obtained using the noise operators is also identical to the correlation Eq. (29) obtained using the boundary operators. For the present (Raman-EIT) scheme, the parameters correspond to the off- 
resonant weak pump field and strong control field, such that

$$
\Delta,\left|\Omega_{c}\right| \gg \gamma_{\alpha \beta},\left|\Omega_{p}\right|
$$

For these parameters, the excited populations and the coherence are negligible $\rho_{d d}^{s t}, \rho_{a a}^{s t}, \widetilde{\rho}_{a b}^{s t} \simeq 0$. In addition, for a negligible mean number of thermal photons and dephasings such that $\gamma_{d b}+\gamma_{b c}=\gamma_{d c}$, the dominant terms in the nonvanishing diffusion coefficients are are simply the normal ordered coefficients

$$
2 \widetilde{D}_{a c, a c}^{n} \simeq 2 \gamma_{a c} \rho_{c c}^{s t} \quad \text { and } \quad 2 \widetilde{D}_{b c, b c}^{n} \simeq 2 \gamma_{b c} \rho_{c c}^{s t},
$$

where $2 \widetilde{D}_{a c, a d}^{n}=2\left(\widetilde{D}_{a d, a c}^{n}\right)^{*} \simeq \gamma_{a d} \widetilde{\rho}_{d c}^{s t}$ are neglected since they are about more than ten times smaller. Hence, the spectral function Eq. (41) to the correlation amplitude due to the noise operator can be written as

$$
\begin{aligned}
\Phi(L, \nu) \simeq & \frac{2 \widetilde{D}_{a c, a c}^{n}}{g_{a} g_{s}} \int_{0}^{L}\left[\psi_{a}^{a^{*}}(\xi, \nu) N_{a c}^{*}(\nu)+\psi_{s}^{a^{*}}(\xi, \nu) M_{a c}^{*}(\nu)\right] \\
& \times\left[\psi_{s}^{s}(\xi, \nu) M_{a c}(\nu)+\psi_{a}^{s}(\xi, \nu) N_{a c}(\nu)\right] d z \\
& +\frac{2 \widetilde{D}_{b c, b c}^{n}}{g_{a} g_{s}} \int_{0}^{L}\left[\psi_{a}^{a^{*}}(\xi, \nu) N_{b c}^{*}(\nu)+\psi_{s}^{a^{*}}(\xi, \nu) M_{b c}^{*}(\nu)\right] \\
& \times\left[\psi_{s}^{s}(\xi, \nu) M_{b c}(\nu)+\psi_{a}^{s}(\xi, \nu) N_{b c}(\nu)\right] d z
\end{aligned}
$$

Before we proceed with Eq. (52), note that for short sample $(N \sigma L \ll 2)$ the intensities would be small and we may neglect $\mathcal{I}_{f}^{b}(L)$ in Eq. (29). By taking $\bar{n}_{f}=0$ in Eq. (31) we obtain the correlation due to the boundary operators

$$
G_{a s}^{(2) b} \simeq\left|C_{a} \frac{g_{a}^{*}}{g_{s}} \int_{-\infty}^{\infty} \psi_{a}^{a^{*}}(L, \nu) \psi_{a}^{s}(L, \nu) e^{i \nu \tau} \frac{d \nu}{2 \pi}\right|^{2}
$$

Since $G_{a s}^{(2) b}$ depends only on two (out of four) coefficients, i.e., $\psi_{a}^{a^{*}}$ and $\psi_{a}^{s}$, the sufficient conditions to obtain a correspondence between $G_{a s}^{(2) b}$ in Eq. (53) and $G_{a s}^{(2) n}$ $\simeq\left|\left\langle\hat{N}_{a}(L, \tau) \hat{N}_{s}(L)\right\rangle\right|^{2}$ via Eqs. (52) and (37) (1) are Condition 1 .

$$
\left|M_{a c}(\nu)\right| \ll\left|N_{a c}(\nu)\right| \quad \text { or } \quad\left|\beta_{s}\right| \ll 1 .
$$

(2) Condition 2.

$$
\left|M_{b c}\right| \ll\left|N_{b c}\right|
$$

or

$$
\left|\alpha_{a} \Omega_{c}^{*} \beta_{s}-\alpha_{s} \Omega_{p}\right| \ll\left|\alpha_{s} \Omega_{p} \beta_{a}-\alpha_{a} \Omega_{c}^{*}\right| .
$$

Note that for $\gamma_{b c}=0$, we have $\widetilde{D}_{b c, b c}^{n}=0$ and the condition Eq. (55) that involves $\beta_{a}$ does not apply. An analysis of these conditions is given in Appendix B.

The two conditions allow us to neglect $\left|M_{a c}(\nu)\right|$ and $\left|M_{b c}\right|$ in Eq. (52) which simplifies to

$$
\Phi(L, \nu) \simeq \frac{1}{g_{a} g_{s}} \int_{0}^{L} \Lambda(\nu) \psi_{a}^{a^{*}}(\xi, \nu) \psi_{a}^{s}(\xi, \nu) d z,
$$

where

$$
\begin{aligned}
\Lambda(\nu) & =2 \widetilde{D}_{a c, a c}^{n}\left|N_{a c}(\nu)\right|^{2}+2 \widetilde{D}_{b c, b c}^{n}\left|N_{b c}(\nu)\right|^{2} \\
& \simeq 2 \rho_{c c}^{s t}\left|\alpha_{a}(\nu)\right|^{2}\left(\gamma_{a c}+\gamma_{b c}\left|\frac{\Omega_{c}^{*}}{T_{b c}(\nu)}\right|^{2}\right) .
\end{aligned}
$$

Again, for sufficiently short medium $L q_{ \pm} \gg 1$, the local approximation Eq. (21) holds and by taking $\rho_{c c}^{s t} \simeq 1$ and $\gamma_{b c}$ $=0$ in Eq. (51) we have

$$
\Phi(L, \nu) \simeq \frac{L\left(2 \gamma_{a c}\right)}{g_{s} g_{a}}\left|\alpha_{a}(\nu)\right|^{2} \psi_{a}^{a^{*}}(L, \nu) \psi_{a}^{s}(L, \nu) .
$$

The $\left|\alpha_{a}(\nu)\right|^{2}$ has two symmetric peaks with value $\left|\alpha_{a, \max }\right|^{2}$ that coincide with the peaks of the function antisymmetric function $\psi_{a}^{a^{*}}(L, \nu) \psi_{a}^{s}(L, \nu)$. For large $\Omega_{c}$, we find that $\left|\alpha_{a, \max }\right|^{2} \psi_{a}^{a^{*}}(L, \nu) \psi_{a}^{s}(L, \nu)$ is a good approximation to $\left|\alpha_{a}(\nu)\right|^{2} \psi_{a}^{a^{*}}(L, \nu) \psi_{a}^{s}(L, \nu)$. Therefore we may put $\alpha_{a}(\nu)$ $\simeq g_{a}^{*} \kappa_{a}^{*} / \gamma_{a c}$ in Eq. (58). Finally, from Eqs. (37) and (35) we obtain the noise part of the cross correlation

$$
G_{a s}^{(2) n} \simeq\left|\frac{4 \pi L\left|\kappa_{a}\right|^{2}}{A N \gamma_{a c}} \frac{g_{a}^{*}}{g_{s}} \int_{-\infty}^{\infty} \psi_{a}^{a^{*}}(L, \nu) \psi_{a}^{s}(L, \nu) e^{i \nu \tau} d \nu\right|^{2}
$$

which is identical to Eq. (53), the result using boundary operators.

Thus, under appropriate conditions of (a) negligible populations in the excited levels, (b) vanishing decoherence between the grounds levels, and (c) short sample, Eq. (35) is qualitatively identical to Eq. (29), i.e., the correlation profile obtained using the boundary operators coincide with that using noise operators. As seen from the solutions Eqs. (6), (7), (14), and (15) of the coupled parametric oscillator, in general there is no correspondence between the noise part and the boundary part because of the spatially nonlocal noise coefficients and the fundamental distinction between the noise (atomic) operators and the boundary (field) operators. Thus, the above correspondence is a unique property of the twophoton Raman-EIT amplifier. The correspondence means that the role of quantum noise can be effectively replaced by the field operators at the boundary. Here the noise operators are not needed for correct qualitative description, and this provide a significant simplification in the calculations involving correlations.

\section{B. Conditions for $\operatorname{Re} \beta_{f}, \operatorname{Im} \beta_{f} \ll 1$}

It is not possible to obtain simple analytical constraints for correspondence from the expressions of $\beta_{f}$ in Eqs. (A7) and (A8). We plot the real and imaginary parts of $\beta_{s}$ and $\beta_{a}$ in Fig. 5. We can identify three resonances for $\beta_{s}$ at (a) $+\Omega_{c}$, (b) $-\Omega_{c}$, and (c) $-\Delta$. There are also three resonances for $\beta_{a}$ at (a) $-\Delta+\Omega_{c}$, (b) $-\Delta-\Omega_{c}$, and (c) 0 . A closer look at Fig. 5 (b) reveals that $\operatorname{Re} \beta_{f}$ are "dispersivelike" while $\operatorname{Im} \beta_{f}$ are "absorptivelike."

For sufficiently large detuning $\left(|\Delta| \geqslant 30 \gamma_{a c}\right)$ and large field $\left(\Omega_{c} \gtrsim 10 \gamma_{a c}\right)$, the peaks of the resonances in Fig. 5(b) are mostly less than 0.1 . Thus, the magnitudes of $\operatorname{Re} \beta_{a}$ and $\operatorname{Im} \beta_{a}$ are negligible when the control field $\Omega_{c}$ and detuning $\Delta$ are sufficiently large even if the decoherence $\gamma_{b c}$ is finite, 


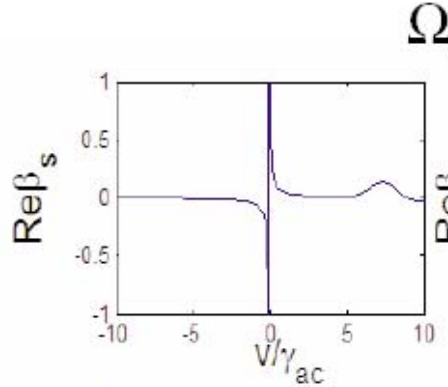

$\Omega_{\mathrm{c}}=\gamma_{\mathrm{ac}}$
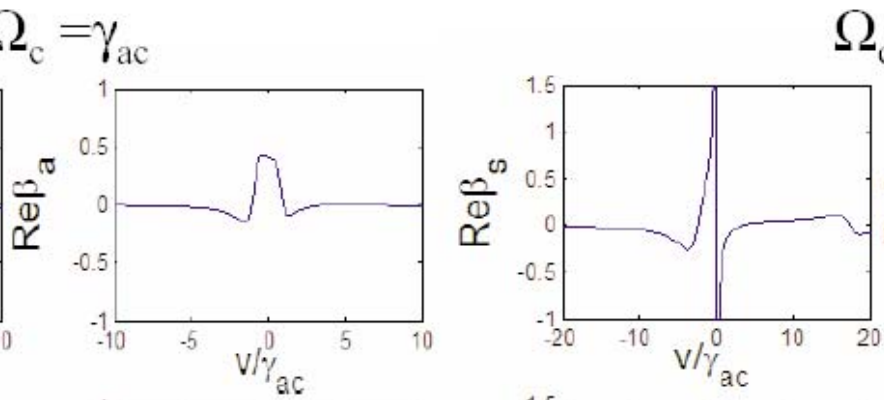

$\Omega_{\mathrm{c}}=10 \gamma_{\mathrm{ac}}$
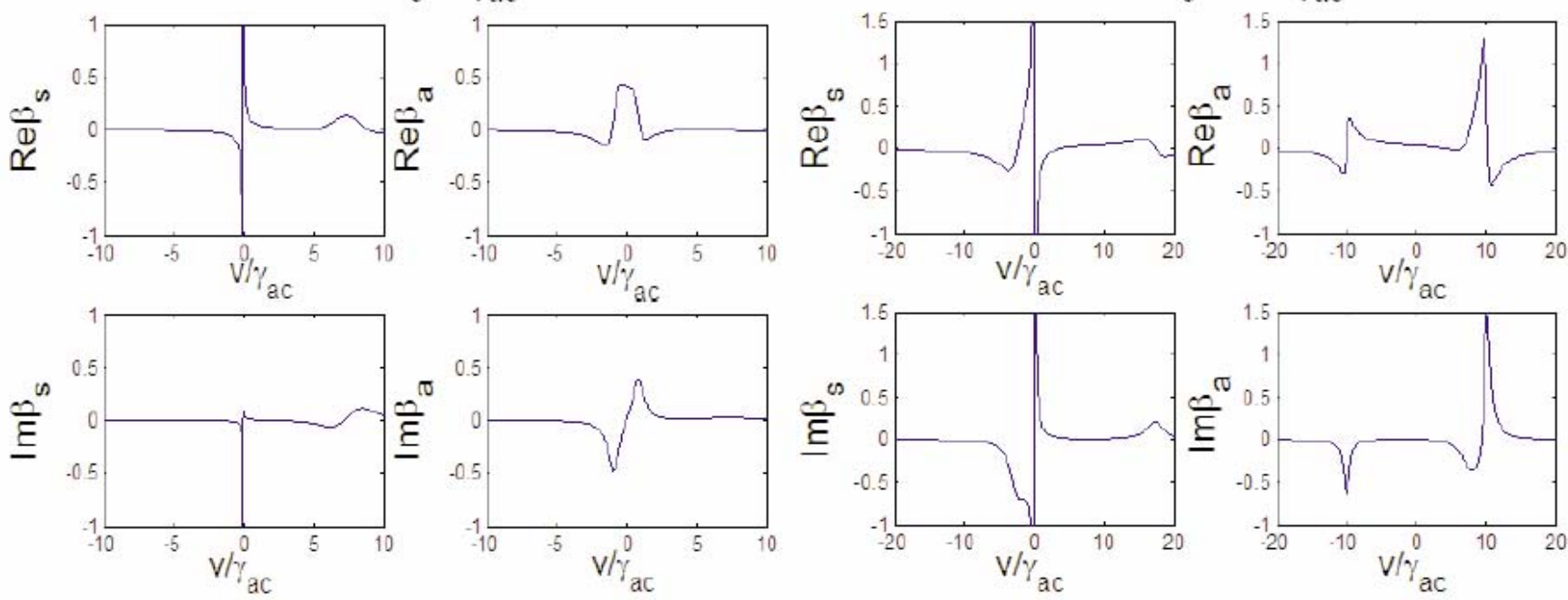

a) $\Delta=-7.5 \gamma_{\mathrm{ac}}, \gamma_{\mathrm{bc}}=0$

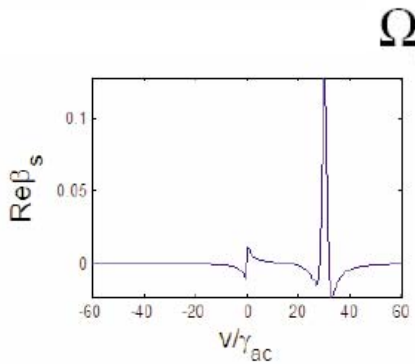

$\Omega_{\mathrm{c}}=\gamma_{\mathrm{ac}}$

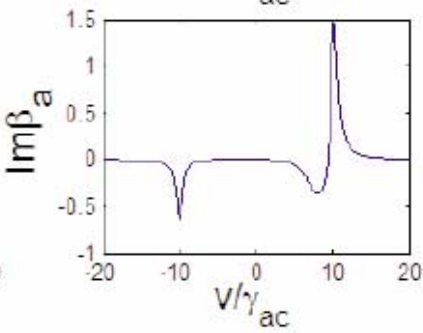

$\Omega_{\mathrm{c}}=10 \gamma_{\mathrm{ac}}$
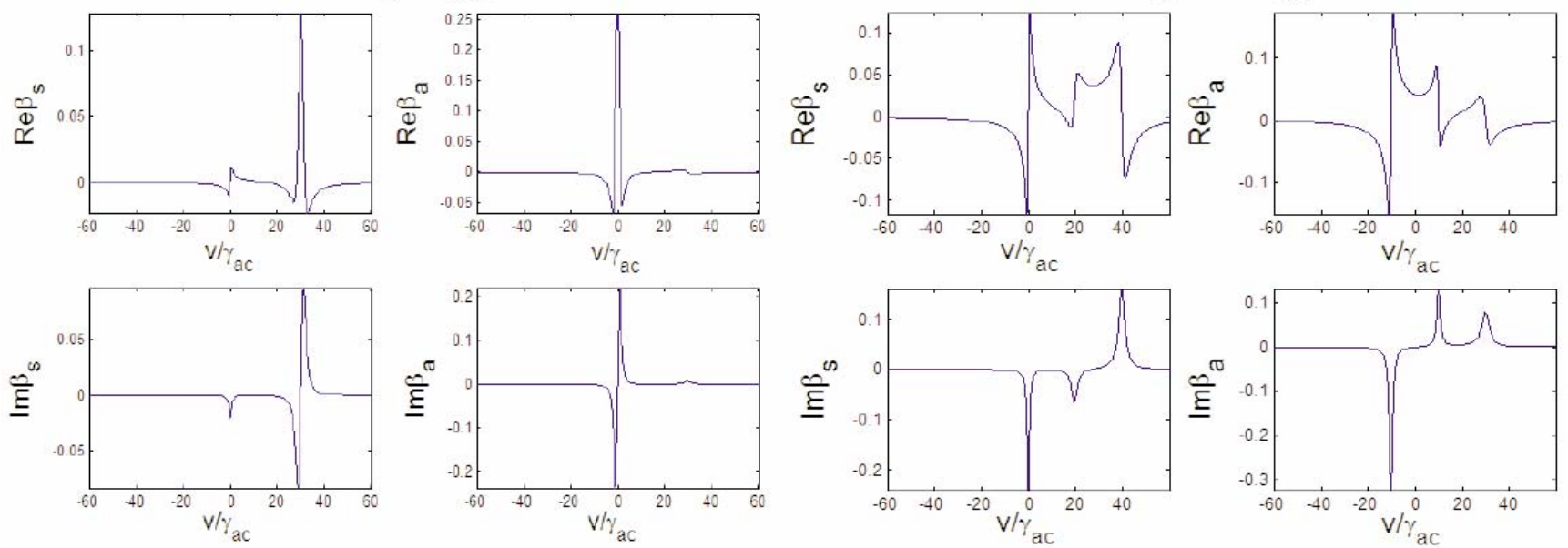

b) $\Delta=-30 \gamma_{\mathrm{ac}}, \gamma_{\mathrm{bc}}=0.6 \gamma_{\mathrm{ac}}$

FIG. 5. (Color online) The real and imaginary parts of $\beta_{s}$ and $\beta_{s}$ as a function of Fourier frequency $\nu$ for (a) $\gamma_{b c}=0.6 \gamma_{a c}, \Delta=-7.5 \gamma_{a c}$ and (b) with decoherence $\gamma_{b c}=0.6 \gamma_{a c}$ and larger detuning $\Delta=-30 \gamma_{a c}$, with $\Omega_{p}=0.4 \gamma_{a c}$.

and it is possible to obtain the correspondence between the results with noise operators and with boundary operators. This is supported by the analysis in Appendix B.

For $\gamma_{b c}=0$ [Fig. 5(a)], there are sharp peaks around $\nu$ $\simeq 0$ in $\beta_{s}$. The $\operatorname{Re} \beta_{s}$ goes like $\wp / \nu$ and $\operatorname{Im} \beta_{s} \sim \delta(\nu)$. Fortunately, both are highly confined around a very narrow region around $\nu \simeq 0$ and fall off rapidly as $|\nu|$ increases. Actually, for $\gamma_{b c}=0$ and $\nu=0$, the expressions of $\beta_{f}$ in Eqs. (A7) and (A8) give $\beta_{s}=\Omega_{c} / \Omega_{p}^{*}, \beta_{a}=\Omega_{p}^{*} / \Omega_{c}$, and $\beta_{s} \beta_{a}=1$. But this particular point gives no contribution to the integration over $\nu$. The effects of finite $\gamma_{b c}$ in Fig. 5(b) are (a) the removal of the narrow peaks and (b) smaller peaks at resonances. Thus, when $\gamma_{b c}=0$ (no decoherence) the correspondence is still possible for the Raman-EIT scheme.

For the parameters $\gamma_{b c} \simeq 0.6 \gamma_{a c}, \Omega_{p}=0.4 \gamma_{a c}, \Delta=-7.5 \gamma_{a c}$ used in the experiment of Balic et al. [2], the peaks in Fig. 5(a) are comparable to 1 , so $\beta_{f}$ cannot be neglected. The detuning $\Delta$ is not large enough to satisfy Eq. (50) for the Raman-EIT scheme. So, the noise operators are required to obtain agreement with their results, as done in Ref. [1].

\section{Identical fields for Raman-EIT and short sample}

At this point, we are ready to proceed from Sec. III E to show the correspondence between Eqs. (6) and (7) and Eqs. (14) and (15) for the Raman-EIT scheme. Under the conditions that give $\operatorname{Re} \beta_{f}, \operatorname{Im} \beta_{f} \ll 1$ (as discussed in in Sec. V B), the commutations Eq. (C3) simplify to

$$
\left[\bar{F}_{f}(z, \nu), \bar{F}_{f}^{\dagger}\left(z^{\prime}, \nu^{\prime}\right)\right] \simeq\left[\hat{F}_{f}(z, \nu), \hat{F}_{f}^{\dagger}(z, \nu)\right] .
$$

Thus, Eqs. (C4) and (C5) give $\bar{C}_{s}(z, \nu) \simeq \mathcal{C}_{s}(z, \nu)$ and the coefficient in Eq. (25) becomes $\bar{C}_{f}^{\text {int }}(\nu) \simeq \int_{0}^{L} \mathcal{C}_{f}(z, \nu) d z$. 
Equation (51) simplifies the coefficients [Eqs. (C8) and (C9)] for the commutation relations Eq. (C7) to

$$
\begin{gathered}
\mathcal{C}_{s}(\nu) \simeq-\frac{(2 \pi)^{3}}{A N}\left|\alpha_{s}(\nu) \frac{\Omega_{p}}{T_{b c}(\nu)}\right|^{2} 2 \gamma_{b c} \rho_{c c}^{s t}, \\
\mathcal{C}_{a}(\nu) \simeq \frac{(2 \pi)^{3}}{A N}\left|\alpha_{a}(\nu)\right|^{2} 2\left\{\gamma_{a c}+\gamma_{b c}\left|\frac{\Omega_{c}}{T_{b c}(\nu)}\right|^{2}\right\} \rho_{c c}^{s t} .
\end{gathered}
$$

For $\gamma_{b c}=0$, Eq. (62) gives the coefficient in Eq. (25) as

$$
\overline{\mathcal{C}}_{a}^{i n t}(\nu) \simeq \frac{L(2 \pi)^{3}}{A N}\left|\alpha_{a}(\nu)\right|^{2} 2 \gamma_{a c} \rho_{c c}^{s t} .
$$

As shown in the discussion below Eq. (58), the $\nu$ dependence in $\alpha_{s}(\nu)$ is immaterial for the Raman-EIT scheme. Thus $\bar{C}_{a}^{i n t}$ becomes effectively like the constant $C_{f}$ in the commutation relation Eq. (20) for the boundary operators. This shows the correspondence between the noise part $\left(\hat{N}_{f}\right)$ of the fields and the boundary operator part $\left(\hat{B}_{f}\right)$.

\section{Ratio of noise correlation amplitude to boundary correlation amplitude}

By comparing $g_{a s}^{(2) n}$ and $g_{s a}^{(2)}$ the results obtained with noise operators only and those obtained with both boundary and noise operators in Figs. 3 and 4, we find that the contribution of the input boundary operators is small compared to the contribution of the noise operators. This can be verified analytically from Eqs. (59) and (53), which give the approximate ratio $G_{a s}^{(2) n} / G_{a s}^{(2) b} \simeq\left|\frac{3}{2} \lambda_{a}^{2} N L \frac{\Gamma_{a}}{\gamma_{a c}}\right|^{2}$ for the Raman-EIT scheme, where $\Gamma_{a}=\frac{\wp^{2} v_{a}^{3}}{3 \pi \varepsilon_{0} \hbar c^{3}}$ and $\lambda_{a}=2 \pi c / \nu_{a}$.

The ratio can be obtained in a different way. By using expressions for the two-photon amplitude in Sec. IV and inserting $2 \widetilde{D}^{n} \simeq 2 \gamma_{a c}, \quad \max \left\{M_{x}, N_{x}\right\} \sim \alpha_{a} \sim g_{a} N_{\wp c \mu_{o} \nu_{a}} / 2 \gamma_{a c}$, and neglecting the Stark shift $\delta_{a}$, we find the ratio of the correlation amplitudes of the noise operators to that of the boundary operators for the Raman-EIT scheme as

$$
\begin{aligned}
r & =\frac{\left\langle\hat{N}_{a}(L, t+\tau) \hat{N}_{s}(L, t)\right\rangle}{\left\langle\hat{B}_{a}(L, t+\tau) \hat{B}_{s}(L, t)\right\rangle} \\
& \sim \frac{(2 \pi / A N)\left(2 \widetilde{D}^{n}\right)\left(\alpha_{a} / g_{a}\right)^{2} L}{\pi \hbar \nu_{f} /\left(\varepsilon_{o} c A\right)} \\
& =N \lambda_{a}^{2} L \frac{3 \Gamma_{a}}{2 \gamma_{a c}},
\end{aligned}
$$

which is the same as the result using Eqs. (59) and (53).

By using an amplifier length of $1 \mathrm{~mm}, \lambda \sim 400 \mathrm{~nm}$, and dilute gas density $N \sim 10^{17} \mathrm{~m}^{-3}$, we find $r \sim 50$. This shows that the contribution of the boundary operators is small compared with the noise operators. This can be easily understood from the fact that the noise operators account for the quantum fluctuations in the entire medium while the boundary operators include only the quantum fluctuations at the input boundary. Equations (35)-(44) imply that the amplified quantum fluctuations contribute to the two-photon correla- tion via the diffusion coefficients. This shows the importance of the atom-fields fluctuations to the nonclassical two-photon correlations.

It is interesting to note that if a shorter transition wavelength (such as ultraviolet) were used in the atomic scheme, $r \sim 1$ and the terms due to the boundary operators would be significant. Here, we have considered the propagation along the $z$ axis in a thin cylindrical medium with Fresnel number $F=A / \lambda L \lesssim 1$, where the transverse effects such as diffraction can be neglected.

\section{DISCUSSIONS}

Noise vs. no noise. In Fig. 2, we compare the correlations for (i) single atom [16], (ii) without noise operators (from Sec. IV B), and (iii) with noise operators only (from Sec. IV C). For an optically thin sample and in the absence of decoherence $\gamma_{b c}$, the results for the three cases are in good qualitative agreement. However, quantitative agreement (in normalized $g_{a s}^{(2)}$ ) is obtained when $\Omega_{c}$ is sufficiently large (also see Fig. 3). When $\gamma_{b c}$ is finite, the results with and without noise do not agree, especially when $\Omega_{c}$ is small. Since the noise part (and not the boundary part) gives the antibunching, here, we may say that the quantum fluctuations of the atomic ensembles are responsible for the nonclassical effect of antibunching. Also, qualitative agreement is obtained only for sufficiently large $\Omega_{c}$. A similar trend is found in an optically thick sample (see Figs. 3 and 4).

Physics behind boundary and noise operators. The boundary operators $\hat{E}_{f}(0, \nu)$ are characterized by the quantum fluctuations of vacuum fields at the input boundary of the medium, while the noise operators $\hat{F}_{x}$ are determined by the quantum fluctuations of atoms and vacuum fields in the entire medium. The source of fluctuations in the medium is not simply vacuum noise of the form $\sum_{\mathbf{k}} g_{\mathbf{k}}^{j} \hat{a}_{\mathbf{k}}(0) e^{i \mathbf{k} \cdot \mathbf{r}} e^{-i \nu_{\mathbf{k}} t}$ but also characterized largely by a combined vacuum noise [via $\left.\hat{a}_{\mathbf{k}}(0)\right]$ and atomic noise [via $\left.\hat{\sigma}_{x y}(0)\right]$ associated with the atomic populations and decoherences. Thus, for sufficiently large optical density the effects of $\hat{F}_{x}$ would become important and the correlation at the output medium may differ substantially from that of a thin medium or single atom case.

Fluctuation-dissipation and vacuum fluctuations. When one goes through the derivation of the quantum Langevin equations, one sees that they contain atomic fluctuations (via the noise operators) and dissipations (via decay rates and decoherences) due to the vacuum fluctuations of the fields. The noise parts $\hat{N}_{f}$ contain fluctuations and dissipations of the atoms, but the boundary parts $\hat{B}_{f}$ describe only atomic dissipations (via $\psi_{f}^{g}$ ) and vacuum fluctuations. Thus, there is no question that both are complementary and necessary to provide the ultimate truthful description. One might argue that the theory without noise is inconsistent with the fluctuation-dissipation theorem and therefore would never yield the correct result. Whenever the boundary operators give results that agree qualitatively with the noise operators, they provide the same underlying physics to the correlation as the quantum noise. Here, the semiclassical theory with only boundary parts is a sufficient substitute to the noise 

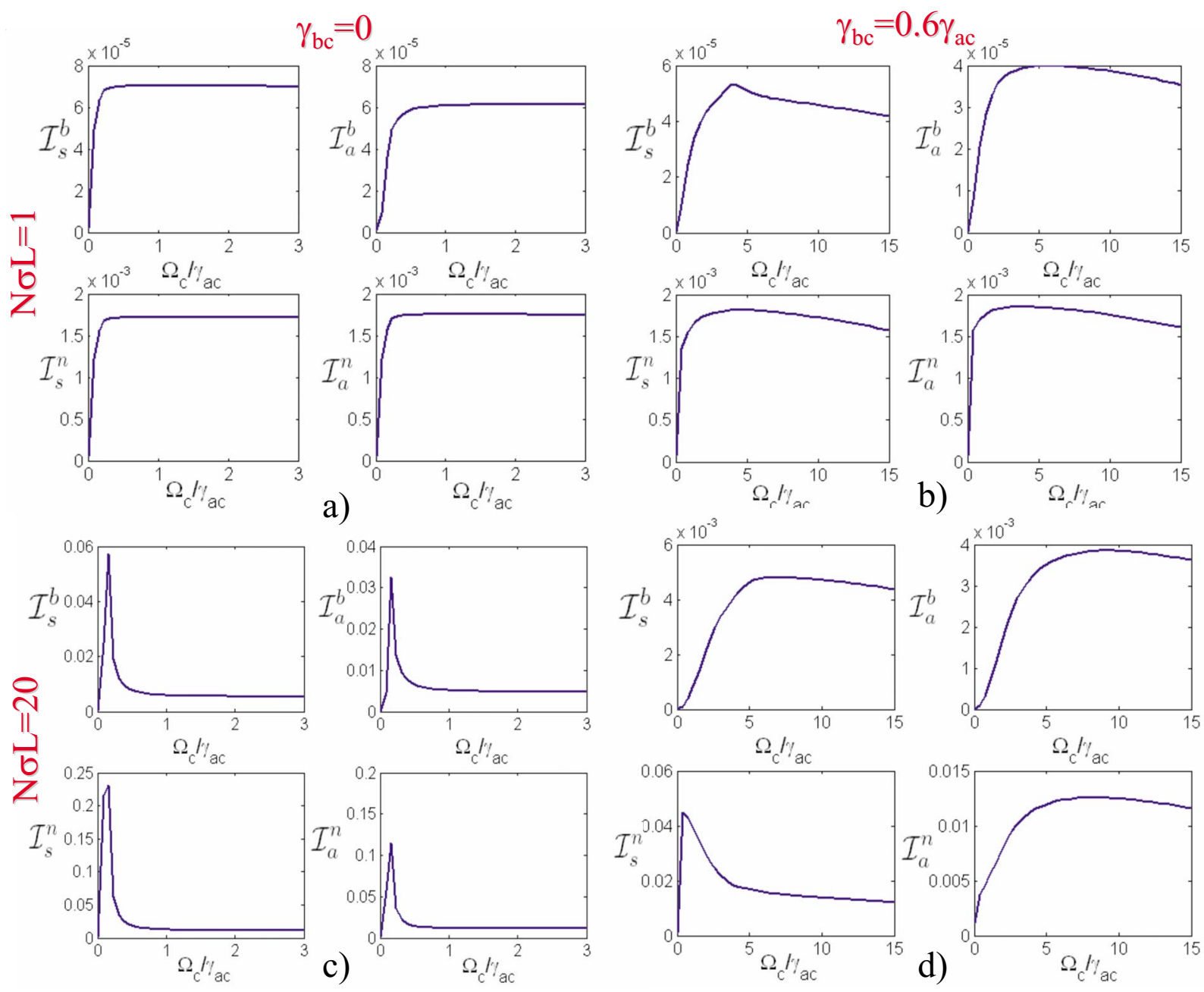

FIG. 6. (Color online) Intensities of the Stokes and anti-Stokes obtained with noise operators $\mathcal{I}_{s}^{n}, \mathcal{I}_{a}^{n}$ and with boundary operators $\mathcal{I}_{s}^{b}, \mathcal{I}_{a}^{b}$ for forward geometry. (a) Small optical density $N \sigma L=1$ and without decoherence $\gamma_{b c}=0$. (b) Small optical density and with decoherence. (c) Large optical density and without decoherence. (d) Large optical density and with decoherence. Other parameters are $\Delta=-7.5 \gamma_{a c}$ and $\Omega_{p}$ $=0.4 \gamma_{a c}$. The intensities without noise operators give quite good qualitative results for zero decoherence even if the optical density is large. For small optical density, the intensities increase rapidly with control field but saturate at a small field. In contrast, for large optical density the intensities drop rapidly before saturation. The onset of saturation is larger when $\gamma_{b c}$ is finite.

parts $\hat{N}_{f}$ which can be neglected. However, this does not mean that there are no fluctuations which always accompany dissipations, in accordance with the fluctuation-dissipation theorem. In other words, sufficient physics can be obtained without the noise operators when the atomic fluctuations in the medium and the vacuum fluctuations give essentially the same effects to the correlation, as in the case of zero decoherence $\gamma_{b c}=0$, large $\Omega_{c}$, and small optical density $(N \sigma L$ $\ll 1)$.

Semiclassical or quantum. In the commutation relation (20) for the input boundary operators, note that quantum mechanics enters through $\hbar$ in $C_{f}$ and quantum electrodynamics (QED) via the bosonic commutation relation. Therefore, the solutions of the boundary parts alone can be considered as a QED theory instead of a semiclassical theory. However, the boundary parts describe only the quantum fluctuations of the vacuum fields (in the absence of external inputs) and not the fluctuations in the atomic medium. Thus, solutions based on the boundary parts alone are incomplete QED theory.
Effects of decoherence $\gamma_{b c}$. Unlike the coherences with dipole-allowed transitions $\gamma_{a c}, \gamma_{d c}, \gamma_{a b}, \gamma_{d b}$, and $\gamma_{a d}$, the origin of the decoherence $\gamma_{b c}$ is not due to dissipation. But it contributes to the atom-field fluctuations and therefore, can significantly affect the correspondence between the correlations with the boundary operators and those with the noise operators.

The decoherence $\gamma_{b c}$ and $\Omega_{c}$ have opposite effects on the correlation. When the atoms are transferred to level $b$, they need to be pumped to level $a$ fast enough by $\Omega_{c}$ before they are decohered by $\gamma_{b c}$. This explains the smaller correlation for finite $\gamma_{b c}$ and the larger correlation for larger $\Omega_{c}$ in Fig. 2. Thus, a large control field would minimize the effects of atomic fluctuations via $\gamma_{b c}$ and consequently dwarfs the distinctions between the fluctuations of the vacuum fields and the atom fields in the medium. Decoherence $\gamma_{b c}$ also tends to reduce the correlation time between the Stokes and antiStokes (Figs. 2-4), as well as the maximum signals generated via parametric process, i.e., coherent Raman scattering [Figs. 6(c) and 6(d)]. When the pump and control fields are 
comparable [Fig. 6], the amplified quantum fields become classically correlated (bunching) in the presence of decoherence $\gamma_{b c}$. For sufficiently large optical density, the antibunching dip apears at a finite $\tau$. This is due to the appreciable EIT group delay of the anti-stokes when the control field is weak.

Effects of larger dipole moment. In Fig. 3, the parameters are for ${ }^{87} \mathrm{Rb}$ which give a larger transition dipole moment than in Fig. 2. This gives the larger coupling strengths $g_{s}, g_{a}$, and optical thickness $N \sigma L=\frac{\left|\wp_{a c}\right|^{2} v_{a}}{\hbar c \varepsilon_{o} \gamma_{a c}} N L=\left(2 g_{s} \kappa_{s}\right) L / \gamma_{a c}$. The increment of $\left|\wp_{a c}\right|$ has the same effect as $N L$ except that it changes quadratically. The effects are: (1) larger finite correlation at $\tau=0$ and (2) smaller correlation time. For a single particle, $g_{a s}^{(2)}$ would be zero at $\tau=0$. The finite correlation at $\tau=0$ is a feature in common with the resonance fluorescence process of more than one atom [17]. By comparing Figs. 2 and 4 for $g_{a s}^{(2)}$, we see that the larger dipole moment of ${ }^{87} \mathrm{Rb}$ leads to a smaller antibunching slope at $\tau=0$, which is also accompanied by a larger value of $g_{a s}^{(2)}(\tau=0)$ and a smaller peak of $g_{a s}^{(2)}$.

Reverse correlation. The finite value of the reverse correlation $g_{s a}^{(2)}$ in Fig. 4 is a propagation effect of the extended medium. When $\Omega_{c}$ is large, anti-Stokes photons are generated shortly after the emission of Stokes photons. The antiStokes photons are correlated to the Stokes instead of otherwise, so $g_{s a}^{(2)}$ is negligible as shown in Fig. 3.

In extended medium, the anti-Stokes photons may build up (to some extent) into a weak quasicoherent field, referred to as $\Omega_{a}$. When $\Omega_{c}$ is small, it creates (together with $\Omega_{a}$ ) a small coherence $\rho_{b c}$ which is driven by the pump field to produce the Stokes photons. Here, the Stokes follow the antiStokes, giving a finite value of $g_{s a}^{(2)}$. For $\Omega_{c}<\Omega_{p}$, the $g_{s a}^{(2)}$ shows antibunching with the period of oscillations about $\tau_{s a} \sim 2 \pi /|\Delta|$. A dramatic result is found in Fig. 6 for optically thick medium. Here, the reverse correlation with the boundary operators and with the noise operators show distinct contradictory features; antibunching and bunching, respectively. This is the best situaltion for experimental test of the role of noise operators. We also observe that a larger $g_{s a}^{(2)}$ is accompanied by a smaller $g_{a s}^{(2)}$ and vice-versa. The reverse correlation in Fig. 4 should not be taken too seriously because of the small values. For weak control field in Fig. 6, the noise correlation becomes bunching when the optical density increases.

Variations with control field. When $\Omega_{c}$ is weaker than $\Omega_{p}$ the profile of $g_{a s}^{(2)}$ is dominated by exponentially varying functions instead of oscillations (Fig. 4), but antibunching remains. As the control field increases [compare Figs. 2(a) and 2(b)], the nonclassical correlation continues to increase even though the individual Stokes and anti-Stokes intensities become saturated. For $\gamma_{b c}=0$, the onset of saturation is determined by the pump, i.e., $\Omega_{c}^{\text {sat }} \sim \Omega_{p}=0.4 \gamma_{a c}$ [Figs. 6(a) and $6(\mathrm{c})]$. The $\Omega_{c}^{\text {sat }}$ increases in the presence of decoherence [Figs. 6(b) and 6(d)]. This saturation is due to the fact that the intensity of the anti-Stokes is limited by the population in level $b$, which is limited by the weak and largely detuned pump. The intensity of the Stokes is also saturated since it is determined by the coherence $\rho_{b c}$ produced by the control field and the anti-Stokes field.
For large optical density and $\gamma_{b c}=0$ [Figs. 6(c)], both fields are amplified to a maximum far above the saturation level when $\Omega_{c} \sim \Omega_{p} / 2$ and fall off rapidly to a saturated level. This interesting effect is well known and can be used as a switch.

As expected for zero decoherence $\gamma_{b c}=0$, the intensities obtained from the boundary operators are qualitatively correct [Figs. 6(a) and 6(c)]. In the presence of decoherence [Figs. 6(b) and 6(d)] the results without noise operators give no agreement, especially for optically thick medium. Here, the Stokes is amplified only moderately above the saturation level while the anti-Stokes hardly rises beyond the saturation level. The decoherence $\gamma_{b c}$ reduces the contribution of the four-wave mixing process to the fields.

\section{CONCLUSIONS}

We have compared the two-photon correlations obtained using the boundary operators with those using the noise operators. Under certain conditions, the results obtained by neglecting the noise operators can be justified even though this is not valid in general. It is found that the cross correlation obtained with and without noise operators are qualitatively identical only when the optical density is small $N \sigma L<1$ and decoherence $\gamma_{b c}=0$ for forward and backward geometries. This correspondence is a unique property of the parametric amplifier with certain conditions, particularly for the spontaneous Raman-EIT scheme. The correspondence also exists when the decoherence is finite $\gamma_{b c} \neq 0$ provided the control field $\Omega_{c}$ is large. We obtained the ratio $G_{\text {with noise }}^{(2)} / G_{\text {no noise }}^{(2)}$ $\sim\left(N \lambda^{2} L\right)^{2}$ in the absence of external input quantum fields, which shows that the contribution of quantum noise in the medium to the correlation is much larger than the contribution of the vacuum noise via the boundary input operators. We discussed the physics represented by the boundary operators and the noise operators that are useful to explain the correspondence and understand the role of noise operators in connection with the fluctuation-dissipation theorem. We also compared the results with and without the noise operators for the reverse correlation, and the intensities of the Stokes and anti-Stokes fields.

Note added. Recently, a nice paper appeared which includes the noise operators to describe the correlations in the optical fiber [18].

\section{ACKNOWLEDGMENT}

Raymond Ooi would like to thank Professor M. O. Scully for stimulating many discussions.

\section{APPENDIX A: COEFFICIENTS IN COUPLED EQUATIONS}

The coefficients in Eqs. (1)-(3) are given by

$$
\begin{gathered}
\mathcal{G}_{s}=-\frac{\alpha_{s}}{I_{s a}} w_{b b}^{s t}+i\left\{M_{b c} \widetilde{\rho}_{c d}^{s t}-M_{a d} \widetilde{\rho}_{b a}^{s t}\right\}-i \frac{\nu}{c}, \\
\mathcal{G}_{a}=-\frac{\alpha_{a}}{I_{s a}} w_{c c}^{s t}+i\left\{N_{a d} \widetilde{\rho}_{d c}^{s t}-N_{b c} \widetilde{\rho}_{a b}^{s t}\right\}-i \frac{\nu}{c},
\end{gathered}
$$




$$
\begin{aligned}
& \mathcal{K}_{s}=\frac{\alpha_{a} \beta_{s}}{I_{s a}} w_{c c}^{s t}+i\left\{M_{a d} \widetilde{\rho}_{d c}^{s t}-M_{b c} \widetilde{\rho}_{a b}^{s t}\right\} \\
& \mathcal{K}_{a}=\frac{\alpha_{s} \beta_{a}}{I_{s a}} w_{b b}^{s t}+i\left\{N_{b c} \widetilde{\rho}_{c d}^{s t}-N_{a d} \widetilde{\rho}_{b a}^{s t}\right\}
\end{aligned}
$$

with $I_{s a}=1-\beta_{s} \beta_{a}$,

$$
\begin{gathered}
M_{a c}=\frac{i \alpha_{a} \beta_{s}}{I_{s a}}, \quad M_{a d}=\frac{\alpha_{s} \Omega_{c}-\alpha_{a} \Omega_{p}^{*} \beta_{s}}{I_{s a} T_{a d}(\nu)}, \\
M_{b c}=\frac{\alpha_{a} \Omega_{c}^{*} \beta_{s}-\alpha_{s} \Omega_{p}}{I_{s a} T_{b c}(\nu)}, \quad M_{b d}=\frac{i \alpha_{s}}{I_{s a}}, \\
N_{a c}=-\frac{i \alpha_{a}}{I_{s a}}, \quad N_{a d}=\frac{\alpha_{a} \Omega_{p}^{*}-\alpha_{s} \Omega_{c} \beta_{a}}{I_{s a} T_{a d}(\nu)}, \\
N_{b c}=\frac{\alpha_{s} \Omega_{p} \beta_{a}-\alpha_{a} \Omega_{c}^{*}}{I_{s a} T_{b c}(\nu)}, \quad N_{b d}=-\frac{i \alpha_{s} \beta_{a}}{I_{s a}}, \\
\beta_{s}=\frac{\alpha_{s}}{g_{a}^{*} \kappa_{a}^{*}} \Omega_{c} \Omega_{p}\left(\frac{1}{T_{b c}(\nu)}+\frac{1}{T_{a d}(\nu)}\right), \\
\beta_{a}=\frac{\alpha_{a}}{g_{s} \kappa_{s}} \Omega_{c}^{*} \Omega_{p}^{*}\left(\frac{1}{T_{b c}(\nu)}+\frac{1}{T_{a d}(\nu)}\right),
\end{gathered}
$$

and the effective absorption coefficients

$$
\alpha_{s}=\frac{g_{s} \kappa_{s}}{T_{d b}^{*}(\nu)+\delta_{s}}, \quad \alpha_{a}=\frac{g_{a}^{*} \kappa_{a}^{*}}{T_{a c}(\nu)+\delta_{a}},
$$

with the power broadening frequencies

$$
\delta_{s}=\frac{\left|\Omega_{c}\right|^{2}}{T_{a d}(\nu)}+\frac{\left|\Omega_{p}\right|^{2}}{T_{b c}(\nu)}, \quad \delta_{a}=\frac{\left|\Omega_{p}\right|^{2}}{T_{a d}(\nu)}+\frac{\left|\Omega_{c}\right|^{2}}{T_{b c}(\nu)},
$$

the propagation constants $\kappa_{s}=N_{\wp_{b d}} c \mu_{o} \nu_{s} / 2, \quad \kappa_{a}$ $=N \wp_{c a} c \mu_{o} \nu_{a} / 2$, the complex rates $T_{x}(\nu)=T_{x}-i \nu$, and the steady state (superscript " $s t$ ") density matrix elements $\widetilde{\rho}_{\beta \alpha}^{s t}$ $=\left\langle\hat{p}_{\alpha \beta}^{s t}(\infty)\right\rangle$ with $w_{c c}^{s t}=\rho_{a a}^{s t}-\rho_{c c}^{s t}, w_{b b}^{s t}=\rho_{d d}^{s t}-\rho_{b b}^{s t}$.

\section{APPENDIX B: CONDITIONS FOR NOISE-BOUNDARY OPERATORS CORRESPONDENCE}

The two conditions Eqs. (54) and (55) that give the correspondence between the correlation with boundary operators and that with noise operators are elaborated here.

\section{Condition 1}

The condition Eq. (54) can be written as

$$
\left|\frac{T_{a d}(\nu)+T_{b c}(\nu)}{T_{b c}(\nu)\left\{T_{d b}^{*}(\nu) T_{a d}(\nu)+I_{c}\right\}+T_{a d}(\nu) I_{p}}\right|^{2} I_{c} I_{p} \ll 1,
$$

where $I_{p, c}=\left|\Omega_{p, c}\right|^{2}$. We learn from Fig. 5 that the peaks of $\operatorname{Im} \beta_{s}$ occur at $\nu=0,-\Delta \pm \Omega_{c}$. So, we should obtain a collective condition based on the three values of $\nu$.

(i) $\nu=0$. We have

$$
\left|\frac{\Omega_{c} \Omega_{p}\left(\gamma_{a d}+\gamma_{b c}-i \Delta\right)}{\gamma_{b c}\left\{\left(\gamma_{d b}-i \Delta\right)\left(\gamma_{a d}-i \Delta\right)+I_{c}\right\}+I_{p}\left(\gamma_{a d}-i \Delta\right)}\right|^{2} \ll 1 .
$$

By assuming $\gamma_{d b}=\gamma$ and $\gamma_{a d}=2 \gamma$ we obtain the quadratic constraint

$$
\begin{gathered}
{\left[\left(2 \gamma^{2}-\Delta^{2}+I_{c}\right) \gamma_{b c}+I_{p} \gamma\right]^{2}+\Delta^{2}\left(3 \gamma \gamma_{b c}+I_{p}\right)^{2}} \\
-I_{c} I_{p}\left\{\left(2 \gamma+\gamma_{b c}\right)^{2}+\Delta^{2}\right\} \gg 0,
\end{gathered}
$$

with the roots

$$
I_{c}^{(1 \pm)}=-a+\frac{1}{2} b \pm \frac{1}{2} \sqrt{b^{2}-4(a b+c)}
$$

where $a=2 \gamma^{2}-\Delta^{2}+I_{p} \frac{\gamma}{\gamma_{b c}}, \quad b=I_{p}\left[\left(2 \frac{\gamma}{\gamma_{b c}}+1\right)^{2}+\left(\frac{\Delta}{\gamma_{b c}}\right)^{2}\right]$ and $c$ $=\Delta^{2}\left(3 \gamma+\frac{I_{p}}{\gamma_{b c}}\right)^{2}$. By inserting the parameters $\gamma_{b c} \simeq 0.6 \gamma_{a c}, \Omega_{p}$ $=0.4 \gamma_{a c}, \Delta=-7.5 \gamma_{a c}$ used in the experiment of Balic et al. [2], we find that a sufficiently strong control field $\Omega_{c}>10 \gamma$ is required for correspondence.

(ii) $\nu=\left(-\Delta \pm \Omega_{c}\right)$. Here, we have

$$
\beta_{s} \simeq \frac{\Omega_{c} \Omega_{p}\left(-2 \gamma-\gamma_{b c} \pm i 2 \Omega_{c}-i \Delta\right)}{\left[i\left(-\Delta \pm \Omega_{c}\right)-\gamma_{b c}\right]\left(-\gamma \pm i \Omega_{c}\right)\left(-2 \gamma \pm i \Omega_{c}\right)+I_{c}\left[i\left(-\Delta \pm \Omega_{c}\right)-\gamma_{b c}\right]+I_{p}\left(-2 \gamma \pm i \Omega_{c}\right)} .
$$

It is not useful to obtain a lengthy solution for $\left|\beta_{s}\right| \ll 1$. By putting in the above parameters, we find that $\left|\beta_{s}\right| \ll 1$ is satisfied at resonances $-\Delta \pm \Omega_{c}$ for all values of $\Omega_{c}$.

\section{Condition 2}

With the help of the expressions for $\alpha_{f}$ and $\beta_{f}$ in Appen$\operatorname{dix}$ A, the condition Eq. (55) can be expressed as

$$
\left|I_{c p}-T_{a d}(\nu) T_{a c}(\nu)\right|\left|\Omega_{p}\right| \ll\left|I_{c p}+T_{d b}^{*}(\nu) T_{a d}(\nu)\right|\left|\Omega_{c}\right|,
$$

where $I_{c p}=I_{c}-I_{p}$.

From Eq. (50), $\left|\Omega_{p}\right| \ll\left|\Omega_{c}\right|$. So Eq. (B6) also implies $\left|T_{a c}(\nu) \Omega_{p}\right| \ll\left|T_{d b}^{*}(\nu) \Omega_{c}\right|, \quad\left|\gamma_{a c} \Omega_{p}\right| \ll\left|\left(\gamma_{d b}-i \Delta\right) \Omega_{c}\right|, \quad$ or $\quad I_{c}$ $\gg \frac{\gamma_{a c}^{2}}{\left(\gamma_{a b}^{2}+\Delta^{2}\right)} I_{p}$. Since $\frac{\gamma_{a c}^{2}}{\left(\gamma_{a b}^{2}+\Delta^{2}\right)} \ll 1$, the condition $\left|\Omega_{p}\right| \ll\left|\Omega_{c}\right|$, which we already have for the Raman-EIT scheme, is suffi- 
cient. Therefore condition 2 is satisfied by the Raman-EIT scheme.

\section{APPENDIX C: NOISE COMMUTATORS}

The $\bar{F}_{s}$ and $\bar{F}_{a}^{\dagger}$ in Eq. (3) are related to

$$
\begin{aligned}
& \hat{F}_{s}=\alpha_{s}\left[\frac{\Omega_{c}}{T_{a d}(\nu)} G_{a d}-\frac{\Omega_{p}}{T_{b c}(\nu)} G_{b c}+i G_{b d}\right], \\
& \hat{F}_{a}^{\dagger}=\alpha_{a}\left[G_{a d} \frac{\Omega_{p}^{*}}{T_{a d}(\nu)}-G_{b c} \frac{\Omega_{c}^{*}}{T_{b c}(\nu)}-i G_{a c}\right],
\end{aligned}
$$

by $\bar{F}_{s}=\frac{\hat{F}_{s}-\beta_{s} \hat{F}_{a}^{\dagger}}{I_{s a}}, \bar{F}_{a}^{\dagger}=\frac{\hat{F}_{a}^{\dagger}-\beta_{a} \hat{F}_{s}}{I_{s a}}$ (given by Eq. (9) in Ref. [1]). It is straightforward to obtain the relations between the noise commutations

$$
\left\langle\left[\bar{F}_{f}(z, \nu), \bar{F}_{f}^{\dagger}\left(z^{\prime}, \nu^{\prime}\right)\right]\right\rangle=\overline{\mathcal{C}}_{f}(z, \nu) \delta\left(\nu-\nu^{\prime}\right) \delta\left(z-z^{\prime}\right),
$$

where

$$
\begin{aligned}
\overline{\mathcal{C}}_{s}(z, \nu)= & \frac{1}{\left|I_{s a}\right|^{2}}\left[\mathcal{C}_{s}(z, \nu)-\left|\beta_{s}\right|^{2} \mathcal{C}_{a}(z, \nu)-\beta_{s}^{*} \mathcal{C}_{s a}(z, \nu)\right. \\
& \left.-\beta_{s} \mathcal{C}_{s a}^{*}(z, \nu)\right]
\end{aligned}
$$

$$
\begin{aligned}
\overline{\mathcal{C}}_{a}(z, \nu)= & \frac{1}{\left|I_{s a}\right|^{2}}\left[\mathcal{C}_{a}(z, \nu)-\left|\beta_{a}\right|^{2} \mathcal{C}_{s}(z, \nu)-\beta_{a}^{*} \mathcal{C}_{a s}^{*}(z, \nu)\right. \\
& \left.-\beta_{a} \mathcal{C}_{a s}(z, \nu)\right]
\end{aligned}
$$

we have used

$$
\begin{aligned}
& \left\langle\left[\hat{F}_{f}(z, \nu), \hat{F}_{g}\left(z^{\prime}, \nu^{\prime}\right)\right]\right\rangle=\mathcal{C}_{f g}(z, \nu) \delta\left(\nu-\nu^{\prime}\right) \delta\left(z-z^{\prime}\right), \\
& \left\langle\left[\hat{F}_{f}(z, \nu), \hat{F}_{f}^{\dagger}\left(z^{\prime}, \nu^{\prime}\right)\right]\right\rangle=\mathcal{C}_{f}(z, \nu) \delta\left(\nu-\nu^{\prime}\right) \delta\left(z-z^{\prime}\right) .
\end{aligned}
$$

As discussed in Sec. V C, the conditions $\left|\beta_{f}\right|^{2} \ll 1$ allow us to evaluate only $\left[\hat{F}_{f}, \hat{F}_{f}^{\dagger}\right]$, where the coefficients are given by

$$
\begin{aligned}
\mathcal{C}_{s}(z, \nu)= & \frac{(2 \pi)^{3}}{A N}\left|\alpha_{s}(\nu)\right|^{2}\left[\mathcal{D}_{b d, b d}^{a n, n}+\left|\frac{\Omega_{c}}{T_{a d}(\nu)}\right|^{2} \mathcal{D}_{a d, a d}^{a n, n}+\left|\frac{\Omega_{p}}{T_{b c}(\nu)}\right|^{2} \mathcal{D}_{b c, b c}^{a n, n}+\frac{i \Omega_{p}}{T_{b c}(\nu)} \mathcal{D}_{b c, b d}^{a n, n}\right. \\
& \left.-\frac{i \Omega_{p}^{*}}{T_{b c}^{*}(\nu)} \mathcal{D}_{b d, b c}^{a n, n}+\frac{i \Omega_{c}^{*}}{T_{a d}^{*}(\nu)} \mathcal{D}_{b d, a d}^{a n, n}-\frac{i \Omega_{c}}{T_{a d}(\nu)} \mathcal{D}_{a d, b d}^{a n, n}\right]
\end{aligned}
$$

where $\mathcal{D}_{x, x^{\prime}}^{a n, n}(z, \nu)=2 \widetilde{D}_{x, x^{\prime}}^{a n}(z, \nu)-2 \widetilde{D}_{x^{\prime}, x}^{n}(z, \nu)$ depend on the normal (superscript " $n$ ") and antinormal ordered (superscript "an") diffusion coefficients obtained from Einstein's relation. Similarly,

$$
\begin{aligned}
\mathcal{C}_{a}(z, \nu)= & \frac{(2 \pi)^{3}}{A N}\left|\alpha_{a}(\nu)\right|^{2}\left[\mathcal{D}_{a c, a c}^{n, a n}+\left|\frac{\Omega_{p}}{T_{a d}(\nu)}\right|^{2} \mathcal{D}_{a d, a d}^{n, a n}+\left|\frac{\Omega_{c}}{T_{b c}(\nu)}\right|^{2} \mathcal{D}_{b c, b c}^{n, a n}+\frac{i \Omega_{p}^{*}}{T_{a d}(\nu)} \mathcal{D}_{a c, a d}^{n, a n}\right. \\
& \left.-\frac{i \Omega_{p}}{T_{a d}^{*}(\nu)} \mathcal{D}_{a d, a c}^{n, a n}+\frac{i \Omega_{c}}{T_{b c}^{*}(\nu)} \mathcal{D}_{b c, a c}^{n, a n}-\frac{i \Omega_{c}^{*}}{T_{b c}(\nu)} \mathcal{D}_{a c, b c}^{n, a n}\right]
\end{aligned}
$$

where $\mathcal{D}_{x, x^{\prime}}^{n, a n}=2 \widetilde{D}_{x, x^{\prime}}^{n}-2 \widetilde{D}_{x^{\prime}, x^{\prime}}^{a n}$

[1] C. H. Raymond Ooi, Q. Sun, M. S. Zubairy, and M. O. Scully, Phys. Rev. A 75, 013820 (2007).

[2] V. Balic, D. A. Braje, P. Kolchin, G. Y. Yin, and S. E. Harris, Phys. Rev. Lett. 94, 183601 (2005).

[3] L. Mandel and E. Wolf, Optical Coherence and Quantum Optics (Cambridge University Press, Cambridge, 1995).

[4] M. H. Rubin, David N. Klyshko, Y. H. Shih, and A. V. Sergienko, Phys. Rev. A 50, 5122 (1994); R. Andrews, A. T. Joseph, E. R. Pike, and S. Sarkar, J. Opt. B: Quantum Semiclassical Opt. 7, S480 (2005).

[5] J. Wen and M. H. Rubin, Phys. Rev. A 70, 063806 (2004); 74, 023808 (2006); 74, 023809 (2006).

[6] J. Chen, X. Li, and P. Kumar, Phys. Rev. A 72, 033801 (2005); See also Ref. [18].

[7] M. O. Scully and M. S. Zubairy, Quantum Optics (Cambridge University Press, Cambridge, 1997).
[8] M. Hillery, Phys. Rev. A 36, 3796 (1987).

[9] M. Lax, Phys. Rev. 145, 110 (1966).

[10] C. M. Caves, Phys. Rev. D 26, 1817 (1982).

[11] D. F. Walls and G. J. Milburn, Quantum Optics (SpringerVerlag, Berlin, 1994).

[12] C. W. Gardiner and P. Zoller, Quantum Noise, 2nd ed. (Springer, Berlin, 2000).

[13] The derivation of the noise correlation $\left\langle\hat{N}_{a}(t+\tau) \hat{N}_{s}(t)\right\rangle$, is given in the Appendix C in Ref. [1].

[14] D. A. Braje, V. Balic, S. Goda, G. Y. Yin, and S. E. Harris, Phys. Rev. Lett. 93, 183601 (2004).

[15] For backward geometry treated in paper I, the functional forms of $\bar{\psi}_{a}^{s}(L, \nu)$, and $\bar{\psi}_{a}^{a}(L, \nu)$, do not coincide with $\bar{U}_{a}^{s}(\xi, \nu)$ and $\bar{U}_{s}^{a}(\xi, \nu)$, respectively, when $\beta_{f} \rightarrow 0$. However, for sufficiently short sample $\bar{q}_{ \pm} L \ll 1$, such that $\xi=L-z$, may be replaced by $L$, the expansion of $\bar{\Xi}_{\bar{q}}$, and $\bar{\Xi}$, reveals that they actually coincide 
and this has been verified numerically. Please to note that the results for backward geometry are hardly distinguishable from the results for forward geometry in Fig. 3.

[16] M. O. Scully and C. H. Raymond Ooi, J. Opt. B: Quantum Semiclassical Opt. 6, S816 (2004).
[17] H. J. Kimble, M. Dagenais, and L. Mandel, Phys. Rev. Lett. 39, 691 (1977); G. S. Agarwal, A. C. Brown, L. M. Narducci, and G. Vetri, Phys. Rev. A 15, 1613 (1977).

[18] Q. Lin, F. Yaman, and G. P. Agarwal, Phys. Rev. A 75, 023803 (2007). 